\title{
On Runge-Kutta Methods for Parabolic Problems with Time-Dependent Coefficients
}

\author{
By Ohannes A. Karakashian
}

\begin{abstract}
Galerkin fully discrete approximations for parabolic equations with time-dependent coefficients are analyzed. The schemes are based on implicit Runge-Kutta methods, and are coupled with preconditioned iterative methods to approximately solve the resulting systems of linear equations. It is shown that for certain classes of Runge-Kutta methods, the fully discrete equations exhibit parallel features that can be exploited to reduce the final execution time to that of a low-order method.
\end{abstract}

1. Introduction. In this paper we shall analyze fully discrete Galerkin type approximations to solutions of the following initial-boundary value problem: Let $\Omega$ be a bounded domain in $\mathbf{R}^{N}$ with smooth boundary $\partial \Omega$. A real-valued function $u$ is sought, satisfying

$$
\begin{cases}u_{t}=-L(t) u \equiv \sum_{i, j=1}^{N} \frac{\partial}{\partial x_{i}}\left(l_{i j}(x, t) \frac{\partial u}{\partial x_{j}}\right)-l_{0}(x, t) u \quad \text { in } \Omega \times\left(0, t^{*}\right] \\ u(x, t)=0 & \text { on } \partial \Omega \times\left[0, t^{*}\right] \\ u(x, 0)=u^{0}(x) & \text { in } \Omega ;\end{cases}
$$

$\left\{l_{i j}\right\}$ is symmetric, uniformly positive definite, and $l_{0} \geqslant 0$ on $\bar{\Omega} \times\left[0, t^{*}\right]$. In order to guarantee the convergence results below, we shall assume that $l_{i j}, l_{0}$ are sufficiently smooth, and $u^{0}$ is sufficiently smooth and compatible so that $u$ has the required regularity.

In [11], Crouzeix analyzed fully discrete approximations to $u$ that are based on Runge-Kutta methods. His methods require, however, the solution of linear systems with different coefficient matrices at each time step. We shall call this the base scheme. An efficient procedure applied by Douglas, Dupont and Ewing [13] to a Crank-Nicolson discretization of a quasi-linear parabolic equation, consists in using preconditioned iterative methods with a preconditioner that does not change at every step, to approximately solve the underlying base scheme. In [5] Bramble and Sammon used similar iterative methods in their analysis of higher-order (3rd and 4th) single-step fully discrete approximations.

These iterative methods require the stability and consistency estimates to hold in a special norm induced by the preconditioning operator or equivalents thereof. We obtain stability and convergence results for the base scheme in such an appropriate

Received December 21, 1984; revised September 5, 1985.

1980 Mathematics Subject Classification. Primary 65M15; Secondary 65N30. 
norm without requiring stronger regularity assumptions on $u$. We construct and analyze preconditioned iterative methods to approximately solve the linear systems that are efficient and preserve the order of convergence of the schemes. We specifically consider two such schemes. For the first, at every step, using extrapolation of previous values, we obtain an initial approximation which is within the global order of accuracy of the method, which is then refined an additional $O(k)$ by a preconditioned iterative method. This scheme requires $O\left(\ln k^{-1}\right)$ iterations per step, where $k$ is the step size.

Our second scheme requires the use of Runge-Kutta methods which are dissipative in a certain sense. For these schemes, it can be shown that the average work per step is independent of $k$. This is achieved by using initial approximations which are within the local order of accuracy of the method. This scheme is similar to Algorithm 2 of Bramble and Sammon [5]. See also [13].

Furthermore, we show that for a specific class of Runge-Kutta methods, the system relating the intermediate values decouples, whereas all these values can be solved simultaneously, i.e., in parallel. One such family of methods is given by the (real) collocation methods whose corresponding rational functions have distinct real poles. In [4] it is shown that this family of (arbitrarily high-order) methods has the required $A_{0}$-stability property, provided the poles are chosen between 0 and -2 . For such methods, the rate of convergence is $q+1$, where $q$ is the number of intermediate values.

Two additional points worth mentioning are that under certain conditions the fully discrete approximations exhibit decay (as does $u(t)$ ); moreover, no use is made of inverse assumptions.

The paper is organized as follows: In Section two we set the notation and list the spatial approximation results to be used. Section three is devoted to the description of the Runge-Kutta methods and their required properties. In Section four we prove stability and convergence of the base scheme. In Section five, we construct and analyze two variants of the base scheme obtained by approximately solving the linear systems by a preconditioned iterative method. We also show that if the rational function corresponding to the Runge-Kutta method has distinct poles, then both variants exhibit parallel features.

2. Notation and Preliminaries. For $m \geqslant 0$ integer, let $H^{m}$ denote the Sobolev space of real-valued functions defined on $\Omega$. For $m=0$, let $(\cdot, \cdot)$ and $\|\cdot\|$ denote the inner product and norm on $L^{2}=H^{0}$. Also, let $H_{0}^{1}$ denote the subspace of $H^{1}$ consisting of functions that vanish on $\partial \Omega$ in the sense of trace.

We assume that $\{L(t)\}_{0 \leqslant t \leqslant t^{*}}$ is a smooth family of selfadjoint, bounded operators from $H^{l+2} \cap H_{0}^{1}$ onto $H^{l}$, for $l \geqslant 0$. Time derivatives $L^{(j)}(t)$ of $L(t)$ are obtained by differentiating its coefficients with respect to $t$. For $0 \leqslant t \leqslant t^{*}$, let $T(t)$ : $L^{2} \rightarrow H^{2} \cap H_{0}^{1}$ be the solution operator of the problem $L \phi=f$ in $\Omega, \phi=0$ on $\partial \Omega$, with $f$ in $L^{2} .\{T(t)\}_{0 \leqslant t \leqslant t^{*}}$ is a smooth family of bounded operators from $H^{l}$ into $H^{l+2} \cap H_{0}^{1}$, with time derivatives $T^{(j)}(t)$.

We shall assume that $u^{0} \in H^{\mu}$ for some $\mu$ to be specified later, and that $u^{0}$ is compatible so that (cf. [14], [17]), for $0 \leqslant t \leqslant t^{*}, u^{(j)}(t) \in H^{\mu-2 j}$ and $\left\|u^{(j)}(t)\right\|_{l} \leqslant$ $c(j, l)\left\|u^{0}\right\|_{\mu}$, for $l \geqslant 0, j \geqslant 0$ such that $l+2 j \leqslant \mu$. 
For $r \geqslant 2$, let $\left\{S_{h}\right\}_{h>0}$ denote a family of finite-dimensional subspaces of $L^{2}$ and assume that there holds, for $v \in H^{l} \cap H_{0}^{1}$,

$$
\left\|P_{0} v-v\right\| \leqslant c h^{\prime}\|v\|_{l}, \quad 2 \leqslant l \leqslant r,
$$

where $P_{0}$ is the $L^{2}$-orthogonal projection operator onto $S_{h}$. We assume that there exists a seminorm $\|\cdot\|_{I}$ on $S_{h}+\left(H^{2} \cap H_{0}^{1}\right)$ which is actually a norm on $S_{h}$. We assume that there exists a smooth family $\left\{T_{h}(t)\right\}: L^{2} \rightarrow S_{h}$ of approximating operators with the following properties:

(i) For each $t \in\left[0, t^{*}\right], T_{h}(t)$ is selfadjoint, positive semidefinite on $L^{2}$ and positive definite on $S_{h}$.

(ii) There exist constants $c(j)$ independent of $h$ such that

$$
\left\|\left(T_{h}^{(j)}(t)-T^{(j)}(t)\right) f\right\|+h\left\|\left(T_{h}^{(j)}(t)-T^{(j)}(t)\right) f\right\|_{I} \leqslant c(j) h^{\prime}\|f\|_{l-2},
$$

$j \geqslant 0,2 \leqslant l \leqslant r, f \in H^{l-2}, 0 \leqslant t \leqslant t^{*}$.

(iii) Denote the inverse of $T_{h}(t)$ on $S_{h}$ by $L_{h}(t)$.

$\left\{L_{h}(t)\right\}$ is a smooth family of symmetric, positive definite operators on $S_{h}$ with

$$
\begin{gathered}
c_{1}\|\phi\|_{1}^{2} \leqslant\|\phi\|_{I}^{2} \leqslant c_{2}\left(L_{h}(t) \phi, \phi\right), \quad 0 \leqslant t \leqslant t^{*}, \phi \in S_{h}, \\
\left|\left(L_{h}^{(j)}(t) \phi, \psi\right)\right| \leqslant \tilde{c}(j)\|\phi\|_{I}\|\psi\|_{I}, \quad j \geqslant 0,0 \leqslant t \leqslant t^{*}, \phi, \psi \in S_{h} .
\end{gathered}
$$

Galerkin methods giving rise to operators $T_{h}(t)$ satisfying the above assumptions include the standard Galerkin method, two methods of Nitsche and the Lagrange multiplier method of Babuška. For the verification of these properties, see [17].

The following two inequalities are direct consequences of (2.3) and (2.4) (cf. [3]),

$$
\begin{gathered}
\left\|T_{h}^{1 / 2}(t) L_{h}^{(j)}(s) T_{h}^{1 / 2}(t)\right\| \leqslant c(j), \quad j \geqslant 0, s, t \in\left[0, t^{*}\right], \\
\left\|L_{h}^{1 / 2}(t) T_{h}^{1 / 2}(s)\right\|+\left\|T_{h}^{1 / 2}(s) L_{h}^{1 / 2}(t)\right\| \leqslant c, \quad s, t \in\left[0, t^{*}\right] .
\end{gathered}
$$

Note that $\|\cdot\|$ is used here to denote the $L^{2}$ operator norm restricted to $S_{h}$.

For $0 \leqslant t \leqslant t^{*}$, let $w(t)=P_{E}(t) u(t)$ denote the "elliptic projection" of $u(t)$, the solution of (1.1), where $P_{E}(t)$ denotes the operator $T_{h}(t) L(t): H^{2} \cap H_{0}^{1} \rightarrow S_{h}$. With $\eta(t)=w(t)-u(t)$, a suitable extension of Proposition 2.1 of [5] gives

$$
\left\|\eta^{(j)}(t)\right\|+h\left\|\eta^{(j)}(t)\right\|_{I} \leqslant c h^{\prime}\left\|u^{0}\right\|_{l+2 j}, \quad j \geqslant 0,2 \leqslant l \leqslant r, s, t \in\left[0, t^{*}\right] .
$$

Note that the case $j=0$ follows directly from (2.2).

Let $k=t^{*} / n^{*}$ be the time step size with $n^{*}$ a positive integer. With $L_{h}^{n}$ denoting $L_{h}\left(t^{n}\right)=L_{h}(n k), 0 \leqslant n \leqslant n^{*}$, for $c>0$ we define the family of norms on $S_{h}$

$$
\|\phi\|_{c, n}^{2}=\|\phi\|^{2}+c\left\|\left(k L_{h}^{n}\right)^{1 / 2} \phi\right\|^{2}, \quad \phi \in S_{h} .
$$

It follows from (2.6) that these norms are equivalent. For $c_{1}, c_{2}>0$,

$$
d_{1}\left(c_{1}, c_{2}\right)\|\| \phi\left\|_{c_{2}, m} \leqslant\right\| \phi\|\|_{c_{1}, n} \leqslant d_{2}\left(c_{1}, c_{2}\right)\|\phi\|_{c_{2}, m}, \quad 0 \leqslant n, m \leqslant n^{*} .
$$

3. The Fully Discrete Approximations. In this paper, we shall consider Implicit Runge-Kutta methods, IRK for short. These methods are characterized by a set of 
constants

$$
\begin{array}{ccc|c}
a_{11} & \cdots & a_{1 q} & \tau_{1} \\
\vdots & & \vdots & \vdots \\
a_{q 1} & \cdots & a_{q q} & \tau_{q} \\
\hline b_{1} & \cdots & b_{q} &
\end{array}
$$

where the integer $q \geqslant 1$ is the number of intermediate stages.

Given an approximation $U_{h}^{n}$ to $u(n k)$, where $u$ is the solution of (1.1), we define $U_{h}^{n+1}$ in the following way:

$$
U_{h}^{n+1}=U_{h}^{n}-k \sum_{i=1}^{q} b_{i} L_{h}\left(t^{n, i}\right) U_{h}^{n, i}
$$

where

$$
U_{h}^{n, i}=U_{h}^{n}-k \sum_{j=1}^{q} a_{i j} L_{h}\left(t^{n, j}\right) U_{h}^{n, j}, \quad i=1, \ldots, q,
$$

and

$$
U_{h}^{0}=\left(I+k \bar{\lambda} L_{h}(0)\right)^{-1} P_{0}(I+k \bar{\lambda} L(0)) u^{0} .
$$

Here $t^{n, j}=t^{n}+k \tau_{j}, j=1, \ldots, q$, and $\bar{\lambda}$ is a positive constant to be appropriately chosen below.

If the array $A,(A)_{i j}=a_{i j}$, is invertible, as will be the case with all the IRK methods considered in this paper, then (3.1) can be written in the form

$$
U_{h}^{n+1}=\left(1-b^{T} A^{-1} e\right) U_{h}^{n}+b^{T} A^{-1} \tilde{U}_{h}^{n},
$$

where $b, e \in R^{q}$ are given by $b=\left(b_{1}, b_{2}, \ldots, b_{q}\right)$ and $e=(1,1, \ldots, 1)$; and $\tilde{U}_{h}^{n} \in \mathscr{S}_{h}$ $\equiv\left(S_{h}\right)^{q}$ is given by $\tilde{U}_{h}^{n}=\left(U_{h}^{n, 1}, \ldots, U_{h}^{n, q}\right)$.

To every IRK method, there corresponds the rational function $r(z)=1-$ $z b^{T}(I+z A)^{-1} e$, representing the map $y^{n+1}=r(k \lambda) y^{n}$ obtained by applying the method to the ODE $y^{\prime}=-\lambda y$. Often it is more convenient to characterize the stability and accuracy properties of the IRK method in terms of $r(z)$.

We shall consider IRK methods which yield (unconditionally) stable and accurate approximations to $u$. Specifically, we consider methods for which

$$
|r(z)| \leqslant 1, \quad z \geqslant 0 \text {. }
$$

Note that (3.4) implies that

$$
1 \geqslant r(z) \geqslant-1+\delta \text { for some } \delta \geqslant 0 \text { and all } z \geqslant 0 .
$$

It is well known that $\|u(t)\|$ is a decreasing function of $t$. It would be preferable then to use methods which transmit this property to the approximations. For such a purpose, the following property is crucial:

$$
\sup _{z \geqslant \gamma>0}|r(z)|<1 \text {. }
$$

Note that since $r(0)=1$, if (3.6) holds, then $\delta>0$.

We shall also consider IRK methods possessing the so-called Algebraic Stability property (cf. [7], [9], [12])

$$
\left\{\begin{array}{l}
b_{i} \geqslant 0 \\
a_{i j} b_{i}+a_{j i} b_{j}-b_{i} b_{j} \quad \text { positive semidefinite. }
\end{array}\right.
$$


This concept of stability is not only stronger than that of (3.4) but also encompasses that of $B$-stability (cf. [12]).

We shall require these methods to satisfy the following two consistency conditions: Let $\nu \geqslant 1$ and $p \geqslant 2$ be the largest integers for which there hold

$$
\begin{gathered}
b^{T} \Upsilon^{\prime} e=\frac{1}{l+1}, \quad 0 \leqslant l \leqslant \nu-1, \\
A \Upsilon^{\prime} e=\frac{1}{l+1} \Upsilon^{l+1} e, \quad 0 \leqslant l \leqslant p-2,
\end{gathered}
$$

where $\Upsilon$ is the diagonal matrix $\Upsilon=\operatorname{diag}\left\{\tau_{1}, \ldots, \tau_{q}\right\}$.

In general, $\nu \leqslant 2 q$ and $p \leqslant q+1$. It also follows from (3.8) and (3.9) that

$$
e^{-z}-r(z)=O\left(z^{\alpha}\right), \quad \alpha \geqslant 2 \text { for }|z| \text { small. }
$$

The following are examples of IRK methods:

(i) Backward Euler

$$
\frac{1}{1} \mid 1, \quad \nu=1, \quad p=2
$$

satisfies (3.7), (3.6) and (3.5) with $\delta=1$.

(ii) Crank-Nicolson

$$
\begin{array}{l|l}
1 / 2 & 1 / 2
\end{array}, \quad \nu=2, \quad p=2
$$

satisfies (3.7), (3.5) (with $\delta=0$ ) but not (3.6).

(iii) Calahan

$$
\begin{array}{cc|c}
\beta & 0 & \beta \\
1-2 \beta & \beta & 1-\beta
\end{array}, \quad \beta=\frac{1}{2}\left(1+\frac{1}{\sqrt{3}}\right) ; \quad \nu=3, \quad p=2
$$

satisfies (3.7) and (3.6).

In the sequel, we shall prove $O\left(k^{\min (\nu, p)}+h^{r}\right)$ rate of convergence estimates for the approximations generated by (3.1)-(3.3) with IRK methods satisfying (3.4), (3.8) and (3.9). We would thus like to work with methods for which $p$ attains its upper bound $q+1$. In this respect, the class of (real) Implicit Runge-Kutta Collocation methods (IRKC) is particularly interesting (cf., e.g., [4]). Arbitrarily high-order $(\nu=p \geqslant 2)$ and stable (in the sense of (3.6)) IRKC methods can be generated as follows: For $q \geqslant 1$ and $\left\{\gamma_{i}\right\}_{i=1}^{q}$ real, let

$$
Q(z)=\prod_{i=1}^{q}\left(1+\gamma_{i} z\right)=\sum_{j=0}^{q} s_{j} z^{j}
$$

where $\left\{s_{j}\right\}_{j=0}^{q}$ are the "symmetric polynomials" in $\gamma_{1}, \gamma_{2}, \ldots, \gamma_{q}$,

$$
s_{0}=1, \quad s_{j}=\sum_{i_{1}<\cdots<i_{j}} \gamma_{i_{1}} \cdots \gamma_{i_{j}} .
$$

We next define the polynomial $N(t)=\sum_{j=0}^{q}(-1)^{j} s_{j} t^{q-j} /(q-j)$ !, and let

$$
P(z)=\sum_{j=0}^{q}(-1)^{q-j} N^{(j)}(1) z^{q-j}, \quad r(z)=P(z) / Q(z) .
$$


Further, assume that the following holds,

$$
\int_{0}^{1} N(t) d t=0
$$

We have

LEMMA 3.1 [4]. For any $q \geqslant 1$, let $r(z)$ be given by (3.11) and suppose that (3.12) holds. Then

(i) If $\gamma_{i}>0, i=1, \ldots, q$, then $N(t)$ has $q$ distinct positive roots $\tau_{1}, \ldots, \tau_{q}$.

(ii) The IRKC method given by $A=\Upsilon V Z V^{-1}, b^{T} V=e^{T} Z$, where $Z=$ $\operatorname{diag}\left\{1, \frac{1}{2}, \ldots, 1 / q\right\}$ and $V$ is the Vandermonde matrix $V_{i j}=\tau_{i}^{j-1}$, satisfies (3.8) and (3.9) with $\nu=p=q+1$.

(iii) If $\gamma_{i} \geqslant \frac{1}{2}$ then (3.4) holds, and (3.6) holds for $q \geqslant 3$. For $q=1$ or $q=2$, (3.6) holds provided $\gamma_{i}>\frac{1}{2}, i=1, \ldots, q$. In fact, the coefficients of $P(z)$ and $Q(z)$ satisfy

$$
\begin{aligned}
& \frac{\left|P_{i}\right|}{Q_{i}} \leqslant 1 \quad \text { for } i=1, \ldots, q, q \geqslant 1 \text { if } \gamma_{i} \geqslant \frac{1}{2} ; \text { and } \\
& \frac{\left|P_{q}\right|}{Q_{q}}<1 \quad \text { for } q \geqslant 3 \text { if } \gamma_{i} \geqslant \frac{1}{2} \text { and } \\
& \frac{\left|P_{q}\right|}{Q_{q}}<1 \quad \text { for } q=1 \text { or } 2 \text { if } \gamma_{i}>\frac{1}{2} .
\end{aligned}
$$

If $\gamma_{1}, \ldots, \gamma_{q}$ are all chosen equal to a common value $\gamma$, then condition (3.12) becomes equivalent to having $\gamma$ satisfy $L_{q}^{1}(1 / \gamma)=0$, where $L_{q}^{1}(x)$ is the Laguerre polynomial of index 1 and degree $q$. We thus retrieve as a special case a class of "restricted" rational functions used in [2] and [15], among others.

It is also known that for IRKC methods, $\gamma_{1}, \ldots, \gamma_{q}$ are the eigenvalues of $A$. Thus, if the $\gamma_{i}$ 's are distinct (in addition to satisfying the conditions of stability and consistency), then we can write $A=S^{-1} \Lambda S$, where $\Lambda=\operatorname{diag}\left\{\gamma_{1}, \ldots, \gamma_{q}\right\}$. In Section 5 we shall show that in this case the equations (3.2) decouple, leading to $q$ independent equations that may be handled simultaneously.

We end this section by exhibiting a family of 2-stage third-order IRKC methods $(\nu=p=3)$. Let $\gamma_{1}, \gamma_{2}$ be two free parameters. The relation (3.12) in this case reduces to

$$
\frac{1}{6}-\frac{1}{2}\left(\gamma_{1}+\gamma_{2}\right)+\gamma_{1} \gamma_{2}=0
$$

and gives $\nu=p=3$. Furthermore, a straightforward computation gives $\tau_{1}=\gamma_{1}+\gamma_{2}$ $+\sqrt{\gamma_{1}^{2}+\gamma_{2}^{2}}, \tau_{2}=\gamma_{1}+\gamma_{2}-\sqrt{\gamma_{1}^{2}+\gamma_{2}^{2}}$

$$
A=\frac{1}{\tau_{2}-\tau_{1}}\left[\begin{array}{cc}
\tau_{1} \tau_{2}-\frac{1}{2} \tau_{1}^{2} & -\frac{1}{2} \tau_{1}^{2} \\
\frac{1}{2} \tau_{2}^{2} & -\tau_{1} \tau_{2}+\frac{1}{2} \tau_{2}^{2}
\end{array}\right], \quad b=\frac{1}{\tau_{2}-\tau_{1}}\left[\begin{array}{c}
\tau_{2}-\frac{1}{2} \\
-\tau_{1}+\frac{1}{2}
\end{array}\right] .
$$

It can be shown that for $\gamma_{1}, \gamma_{2} \geqslant \frac{1}{2}$, this family of methods satisfies (3.7), and (3.6) if, in addition, $\gamma_{1}, \gamma_{2}>\frac{1}{2}$. In a similar manner, arbitrarily high-order $(\nu=p=q+1)$ IRKC methods can be constructed satisfying (3.6). However, it is known that for $\nu \geqslant 2$, (3.7)-(3.9) imply that $\nu=2 q-1$ or $2 q$ (cf., e.g., Theorem 1 in [6]). Thus, for this class of methods, (3.7) cannot hold for $q \geqslant 3$. 
4. Stability and Convergence of the Base Scheme. Letting $\tilde{\mathscr{L}}_{h}^{n}: \mathscr{S}_{h} \rightarrow \mathscr{S}_{h}$ denote the operator $\operatorname{diag}\left\{L_{h}^{n, 1}, \ldots, L_{h}^{n, q}\right\}$, with $L_{h}^{n, j} \equiv L_{h}\left(t^{n, j}\right)$, we can write (3.2) and (3.1) as

$$
\begin{gathered}
\left(I+k A \tilde{\mathscr{L}}_{h}^{n}\right) \tilde{U}_{h}^{n}=e U_{h}^{n}, \\
U_{h}^{n+1} \equiv \mathscr{R}_{h}^{n} U_{h}^{n}=\left\{I-k b^{T} \tilde{\mathscr{L}}_{h}^{n}\left(I+k A \tilde{\mathscr{L}}_{h}^{n}\right)^{-1} e\right\} U_{h}^{n} .
\end{gathered}
$$

Here $A \tilde{\mathscr{L}}_{h}^{n}$ is understood in the sense of composition of operators on $\mathscr{S}_{h}$, with $A$ viewed as an operator on $\left(L^{2}\right)^{q}$. Similarly, we define $\mathscr{L}_{h}^{n}=\operatorname{diag}\left\{L_{h}^{n}, \ldots, L_{h}^{n}\right\}$. Let us also mention that, in the sequel, $\|\cdot\|$ will also denote the norm $\left\{\left\|\phi_{1}\right\|^{2}+\cdots+\left\|\phi_{q}\right\|^{2}\right\}^{1 / 2}$, with $\left(\phi_{1}, \ldots, \phi_{q}\right) \in \mathscr{S}_{h}$, as well as the corresponding operator norm.

We now briefly describe the results of this section. In Proposition 4.1, we establish the invertibility of the operator $\left(I+k A \tilde{\mathscr{L}}_{h}^{n}\right)$ and obtain estimates on norms of various associated operators, provided the eigenvalues of $A$ satisfy $\operatorname{Re} \lambda \geqslant 0$ and $\lambda \neq 0$. This condition will be assumed throughout the paper; in fact, we shall assume in Section 5 that the eigenvalues of $A$ are positive, real. The proof is a simplification of that of Crouzeix. We give it here for the sake of completeness. In Theorem 4.1, we obtain a stability estimate for the operator $\mathscr{R}_{h}^{n}$ in the $\||\cdot| \mid-$-norm, for IRK methods satisfying (3.4) or (3.6). In Theorem 4.2, we obtain an improved stability estimate for methods satisfying a stronger version of (3.7). In Theorem 4.3, we estimate the local truncation error, again in the |||||| -norm, for methods satisfying (3.8) and (3.9). These results, when combined, give the convergence of the approximations generated by the base scheme (3.1)-(3.3).

Proposition 4.1. Suppose the eigenvalues of $A$ satisfy $\operatorname{Re} \lambda \geqslant 0$ and $\lambda \neq 0$. Then

(i) $I+k A \mathscr{L}_{h}^{n}$ is invertible and the following estimate holds:

$$
\left\|\left(k \mathscr{L}_{h}^{n}\right)^{\theta}\left(I+k A \mathscr{L}_{h}^{n}\right)^{-1}\right\| \leqslant c, \quad 0 \leqslant \theta \leqslant 1,
$$

where $\left(\mathscr{L}_{h}^{n}\right)^{\theta}=\operatorname{diag}\left\{\left(L_{h}^{n}\right)^{\theta}, \ldots,\left(L_{h}^{n}\right)^{\theta}\right\}$.

(ii) There exists a $k_{0}>0$ such that for $k \leqslant k_{0}, I+k A \tilde{\mathscr{L}}_{h}^{n}$ is invertible and the following estimate holds:

$$
\left\|\left(I+k A \tilde{\mathscr{L}}_{h}^{n}\right)^{-1}\right\|+\left\|\left(k \mathscr{L}_{h}^{n}\right)^{1 / 2}\left(I+k A \tilde{\mathscr{L}}_{h}^{n}\right)^{-1}\right\| \leqslant c .
$$

Proof. (i) Let $S^{-1} \Lambda S$ be the Jordan decomposition of $A$, with $\Lambda$ lower triangular; then $I+k A \mathscr{L}_{h}^{n}=S^{-1}\left(I+k \Lambda \mathscr{L}_{h}^{n}\right) S$. Let $\Lambda_{s}$ be the $i_{s} \times i_{s}$ block of $\Lambda$ corresponding to the eigenvalue $\lambda_{s}$, and consider the diagonal operator $\mathscr{L}_{h s}^{n}=\operatorname{diag}\left\{L_{h}^{n}, \ldots, L_{h}^{n}\right\}$ on $\left(S_{h}\right)^{i_{s}}$. Now $I+k \Lambda_{s} \mathscr{L}_{h s}^{n}$ is invertible since $L_{h}^{n}$ is symmetric, positive definite, and $\operatorname{Re} \lambda \geqslant 0$. It follows that $I+k A \mathscr{L}_{h}^{n}$ is invertible. Moreover,

$$
\left(\left(I+k \Lambda_{s} \mathscr{L}_{h s}^{n}\right)^{-1}\right)_{i j}=\left\{\begin{array}{l}
0, \quad j>i, \\
\left(-k L_{h}^{n}\right)^{i-j}\left(I+k \lambda_{s} L_{h}^{n}\right)^{-(i-j+1)}, \quad 1 \leqslant j \leqslant i \leqslant i_{s} .
\end{array}\right.
$$

Using a spectral argument, it follows from (4.5), since $\operatorname{Re} \lambda_{s} \geqslant 0$ and $\lambda_{s} \neq 0$, that for $0 \leqslant \theta \leqslant 1,\left\|\left(k \mathscr{L}_{h s}^{n}\right)^{\theta}\left(I+k \Lambda_{s} \mathscr{L}_{h s}^{n}\right)^{-1}\right\| \leqslant c$. This in turn gives (4.3).

(ii) We write $I+k A \tilde{\mathscr{L}}_{h}^{n}=\left(I+k A \mathscr{L}_{h}^{n}\right)(I-E)$, where

$$
E=\left(I+k A \mathscr{L}_{h}^{n}\right)^{-1} k A\left(\mathscr{L}_{h}^{n}-\tilde{\mathscr{L}}_{h}^{n}\right) .
$$


Letting $\mathscr{T}_{h}^{n}=\left(\mathscr{L}_{h}^{n}\right)^{-1}=\operatorname{diag}\left\{T_{h}^{n}, \ldots, T_{h}^{n}\right\}$, we have

$$
\left(\mathscr{L}_{h}^{n}\right)^{1 / 2}\left(I+k A \tilde{\mathscr{L}}_{h}^{n}\right)\left(\mathscr{T}_{h}^{n}\right)^{1 / 2}=\left(I+k A \mathscr{L}_{h}^{n}\right)\left(I-\left(\mathscr{L}_{h}^{n}\right)^{1 / 2} E\left(\mathscr{T}_{h}^{n}\right)^{1 / 2}\right) .
$$

It follows from (2.5) and (4.3) with $\theta=1 / 2$ that

$$
\left\|\left(\mathscr{L}_{h}^{n}\right)^{1 / 2} E\left(\mathscr{T}_{h}^{n}\right)^{1 / 2}\right\| \leqslant c k .
$$

Hence, $I+k A \tilde{\mathscr{L}}_{h}^{n}$ is invertible if $c k<1$. Now, as in the above,

$$
\begin{aligned}
& \left\|\left(k \mathscr{L}_{h}^{n}\right)^{1 / 2}\left(I+k A \tilde{\mathscr{L}}_{h}^{n}\right)^{-1}\right\| \\
& \quad=\left\|\left[I-\left(\mathscr{L}_{h}^{n}\right)^{1 / 2} E\left(\mathscr{T}_{h}^{n}\right)^{1 / 2}\right]^{-1}\left(k \mathscr{L}_{h}^{n}\right)^{1 / 2}\left(I+k A \mathscr{L}_{h}^{n}\right)^{-1}\right\| \leqslant c,
\end{aligned}
$$

from (4.7) and (4.3) with $\theta=1 / 2$. Now note that from (4.8), $\left\|\left(k \mathscr{L}_{h}^{n}\right)^{1 / 2} \phi\right\| \leqslant$ $c\left\|\left(I+k A \tilde{\mathscr{L}}_{h}^{n}\right) \phi\right\|$ for all $\phi \in \mathscr{S}_{h}$. Thus, from (2.6),

$$
\begin{aligned}
\|\phi\|^{2} & =\left(\left(I+k A \tilde{\mathscr{L}}_{h}^{n}\right) \phi, \phi\right)-k\left(A \tilde{\mathscr{L}}_{h}^{n} \phi, \phi\right) \\
& \leqslant\left\|\left(I+k A \tilde{\mathscr{L}}_{h}^{n}\right) \phi\right\|\|\phi\|+c\left\|\left(k \mathscr{L}_{h}^{n}\right)^{1 / 2} \phi\right\|^{2} .
\end{aligned}
$$

Using the arithmetic-geometric mean inequality, we get from the above that $\|\phi\| \leqslant$ $c\left\|\left(I+k A \tilde{\mathscr{L}}_{h}^{n}\right) \phi\right\|$, or $\left\|\left(I+k A \tilde{\mathscr{L}}_{h}^{n}\right)^{-1} \psi\right\| \leqslant c\|\psi\|$, with $\psi \in \mathscr{S}_{h}$. This together with (4.8) gives (4.4).

THEOREM 4.1. Suppose that the eigenvalues of $A$ satisfy $\operatorname{Re} \lambda \geqslant 0, \lambda \neq 0$ and that (3.4) holds. Let $\zeta^{n}, \zeta^{n+1}, \psi^{n+1}$ in $S_{h}$ be given by $\zeta^{n+1}=\mathscr{R}_{h}^{n} \zeta^{n}+\psi^{n+1}$. Then there exists a constant $\tilde{c}$ such that for any $c^{*}>0$,

$$
\begin{aligned}
\left\|\zeta^{n+1}\right\|_{c^{*}, n+1}^{2} \leqslant & (1+\tilde{c} k)\left\|\zeta^{n}\right\|_{c^{*}, n}^{2} \\
& -\frac{\delta}{3}\left(\left[I-r\left(k L_{h}^{n}\right)\right]\left[I+c^{*} k L_{h}^{n}\right] \zeta^{n}, \zeta^{n}\right)+c k^{-1}\left\|\psi^{n+1}\right\|_{c^{*}, n+1}^{2},
\end{aligned}
$$

where $\delta$ is as in (3.5). Moreover, if (3.6) holds and the constant c(1) in (2.5) is sufficiently small, then $\tilde{c}<0$.

Proof. We let $r_{h}^{n}=r\left(k L_{h}^{n}\right)$ and note that $r_{h}^{n}=I-k b^{T} \mathscr{L}_{h}^{n}\left(I+k A \mathscr{L}_{h}^{n}\right)^{-1} e$. We have

$$
\left\|\zeta^{n+1}\right\|^{2} \leqslant\left\|r_{h}^{n} \zeta^{n}\right\|^{2}+2\left\|\mathscr{R}_{h}^{n}-r_{h}^{n}\right\|\left\|\zeta^{n}\right\|\left\|\zeta^{n+1}\right\|+2\left|\left(\psi^{n+1}, \zeta^{n+1}\right)\right| .
$$

From (2.3), (3.4) and (3.10) it follows that there exists $c_{1} \geqslant 0, k_{0}>0$ sufficiently small such that

$$
\left\|r_{h}^{n}\right\|=\max _{\lambda \in \sigma\left(L_{h}^{n}\right)}|r(k \lambda)| \leqslant 1-c_{1} k, \quad 0<k \leqslant k_{0} ;
$$

moreover, $\mathrm{c}_{1}>0$ if (3.6) holds. This implies that $\mathrm{I}+\mathrm{r}_{h}^{n}$ and $I-r_{h}^{n}$ are nonnegative definite. Hence, using (3.5) and (4.11),

$$
\begin{aligned}
\left\|r_{h}^{n} \zeta^{n}\right\|^{2} & =\left(\left(r_{h}^{n}\right)^{2} \zeta^{n}, \zeta^{n}\right)=\left\|\zeta^{n}\right\|^{2}-\left(\left[I+r_{h}^{n}\right]\left[I-r_{h}^{n}\right]^{1 / 2} \zeta^{n},\left[I-r_{h}^{n}\right]^{1 / 2} \zeta^{n}\right) \\
& \leqslant\left\|\zeta^{n}\right\|^{2}-\delta\left(\left(I-r_{h}^{n}\right) \zeta^{n}, \zeta^{n}\right) \\
& \leqslant\left(1-\frac{\delta}{2} c_{1} k\right)\left\|\zeta^{n}\right\|^{2}-\frac{\delta}{2}\left(\left[I-r_{h}^{n}\right] \zeta^{n}, \zeta^{n}\right)
\end{aligned}
$$


Now with $E$ as in (4.6), after lengthy computations,

$$
\begin{aligned}
\mathscr{R}_{h}^{n}-r_{h}^{n}= & b^{T}\left[\left(I+k A \mathscr{L}_{h}^{n}\right)^{-1}\left(k \mathscr{L}_{h}^{n}\right)^{1 / 2}\right]\left[\left(\mathscr{T}_{h}^{n}\right)^{1 / 2}\left(\mathscr{L}_{h}^{n}-\tilde{\mathscr{L}}_{h}^{n}\right)\left(\mathscr{T}_{h}^{n}\right)^{1 / 2}\right] \\
& \times\left[I-\left(\mathscr{L}_{h}^{n}\right)^{1 / 2} E\left(\mathscr{T}_{h}^{n}\right)^{1 / 2}\right]^{-1}\left(k \mathscr{L}_{h}^{n}\right)^{1 / 2}\left(I+k A \mathscr{L}_{h}^{n}\right)^{-1} e .
\end{aligned}
$$

Hence, from (2.5), (4.3), and (4.7), for $k$ sufficiently small,

$$
\left\|\mathscr{R}_{h}^{n}-r_{h}^{n}\right\|+\left\|\left(L_{h}^{n}\right)^{1 / 2}\left(\mathscr{R}_{h}^{n}-r_{h}^{n}\right)\left(T_{h}^{n}\right)^{1 / 2}\right\| \leqslant c_{2} k, \quad c_{2}=c \cdot c(1) .
$$

Using this and (4.12) in (4.10), we get for any $\varepsilon>0$,

$$
\begin{aligned}
\left(1-c_{2} k-\varepsilon k\right)\left\|\zeta^{n+1}\right\|^{2} \leqslant & \left(1-\frac{\delta c_{1}}{2} k+c_{2} k\right)\left\|\zeta^{n}\right\|^{2}-\frac{\delta}{2}\left(\left[I-r_{h}^{n}\right] \zeta^{n}, \zeta^{n}\right) \\
& +\varepsilon^{-1} k^{-1}\left\|\psi^{n+1}\right\|^{2} .
\end{aligned}
$$

Now letting $\tilde{\zeta}^{i}=\left(k L_{h}^{n}\right)^{1 / 2} \zeta^{i}, i=n, n+1$, and $\tilde{\psi}^{n+1}=\left(k L_{h}^{n}\right)^{1 / 2} \psi^{n+1}$, we get exactly as before,

$$
\begin{aligned}
\left(1-c_{2} k-\varepsilon k\right)\left\|\tilde{\zeta}^{n+1}\right\|^{2} \leqslant & \left(1-\frac{\delta c_{1}}{2} k+c_{2} k\right)\left\|\tilde{\zeta}^{n}\right\|^{2} \\
& -\frac{\delta}{2}\left(\left[I-r_{h}^{n}\right] \tilde{\zeta}^{n}, \tilde{\zeta}^{n}\right)+\varepsilon^{-1} k^{-1}\left\|\tilde{\psi}^{n+1}\right\|^{2}
\end{aligned}
$$

(4.9) now follows from the last two inequalities and (2.5). Also, if $\delta>0$ and $c_{2}$ (i.e., $c(1))$ is small, then it is the case that $\tilde{c}<0$.

The constant $\tilde{c}$ in (4.9) is negative provided (3.6) holds and $L_{h}(t)$ changes slowly with $t$. We shall show that this latter requirement can be waived in the case of methods that satisfy a stronger stability condition and are dissipative in the sense that

$$
|r(\infty)|=\left|1-b^{T} A^{-1} e\right|<1 .
$$

THEOREM 4.2. Suppose that the eigenvalues of $A$ satisfy $\operatorname{Re} \lambda \geqslant 0, \lambda \neq 0$ and that (3.7) and (4.15) hold. Suppose in addition that $b_{i}>0, i=1, \ldots, q$. Let $\zeta^{n+1}, \zeta^{n}$, $\psi^{n+1}$ in $S_{h}$ satisfy $\zeta^{n+1}=\mathscr{R}_{h}^{n} \zeta^{n}+\psi^{n+1}$. Then there exist constants $c^{*}, c_{1}>0, \tilde{c}<0$ such that

(4.16) $\left\|\zeta^{n+1}\right\|_{c^{*}, n+1}^{2} \leqslant(1+\tilde{c} k)\left\|\zeta^{n}\right\|_{c^{*}, n}^{2}-c_{1} k\left(L_{h}^{n} \zeta^{n}, \zeta^{n}\right)+c k^{-1}\left\|\psi^{n+1}\right\|_{c^{*}, n+1}^{2}$.

Proof. For simplicity we shall assume that $\psi^{n+1}=0$. Let $\left\{\phi^{i}\right\}_{i=1}^{q}$ in $S_{h}$ be given by

$$
\phi^{i}=\zeta^{n}-k \sum_{j=1}^{q} a_{i j} L_{h}^{n \cdot j} \phi^{j}, \quad i=1, \ldots, q
$$

Then

$$
\zeta^{n+1}=\zeta^{n}-k \sum_{i=1}^{q} b_{i} L_{h}^{n, i} \phi^{i}
$$

Now from (4.17) and (4.18),

$$
\begin{aligned}
\left\|\zeta^{n+1}\right\|^{2} & =\left\|\zeta^{n}\right\|^{2}-2 k \sum_{i=1}^{q} b_{i}\left(\zeta^{n}, L_{h}^{n, i} \phi^{i}\right)+k^{2} \sum_{i, j=1}^{q} b_{i} b_{j}\left(L_{h}^{n, i} \phi^{i}, L_{h}^{n, j} \phi^{j}\right) \\
& =\left\|\zeta^{n}\right\|^{2}-2 k \sum_{i=1}^{q} b_{i}\left(\phi^{i}, L_{h}^{n, i} \phi^{i}\right)-k^{2} \sum_{i, j=1}^{q} m_{i j}\left(L_{h}^{n, i} \phi^{i}, L_{h}^{\prime, j} \phi^{j}\right),
\end{aligned}
$$


where $m_{i j}=a_{i j} b_{i}+a_{j i} b_{j}-b_{i} b_{j}$. Letting $L_{h}^{n, j} \phi^{j}=\sum_{l=1}^{d} c_{j l} \chi_{l}$, where $\left\{\chi_{l}\right\}_{l=1}^{d}$ is an $L^{2}$-orthonormal basis for $S_{h}$, we get

$$
\sum_{i, j=1}^{q} m_{i j}\left(L_{h}^{n, i} \phi^{i}, L_{h}^{n, j} \phi^{j}\right)=\sum_{l=1}^{d} \sum_{i, j=1}^{q} m_{i j} c_{i l} c_{j l} \geqslant 0
$$

Hence,

$$
\left\|\zeta^{n+1}\right\|^{2} \leqslant\left\|\zeta^{n}\right\|^{2}-2 k \sum_{i=1}^{q} b_{i}\left(L_{h}^{n, i} \phi^{i}, \phi^{i}\right) \leqslant\left\|\zeta^{n}\right\|^{2} .
$$

Also, from (4.17) and (4.18) with $a_{i j}^{-1}=\left(A^{-1}\right)_{i j}$,

$$
\begin{aligned}
\left(L_{h}^{n} \zeta^{n+1}, \zeta^{n+1}\right) & =\left(L_{h}^{n} \zeta^{n}, \zeta^{n+1}\right)-k \sum_{i=1}^{q} b_{i}\left(L_{h}^{n, i} \phi^{i}, L_{h}^{n} \zeta^{n+1}\right) \\
& =r(\infty)\left(L_{h}^{n} \zeta^{n}, \zeta^{n+1}\right)+\sum_{i, j=1}^{q} b_{i} a_{i j}^{-1}\left(L_{h}^{n} \phi^{j}, \zeta^{n+1}\right) .
\end{aligned}
$$

Thus, for any $\varepsilon>0$,

$$
\left\{1-\frac{1}{2}|r(\infty)|-\varepsilon\right\}\left(L_{h}^{n} \zeta^{n+1}, \zeta^{n+1}\right) \leqslant \frac{1}{2}|r(\infty)|\left(L_{h}^{n} \zeta^{n}, \zeta^{n}\right)+c \varepsilon^{-1} \sum_{i=1}^{q}\left(L_{h}^{n} \phi^{i}, \phi^{i}\right) .
$$

Now it follows from (2.5) that $\left(L_{h}^{n} \phi^{i}, \phi^{i}\right) \leqslant(1+c k)\left(L_{h}^{n, i} \phi^{i}, \phi^{i}\right)$. Using this together with (4.19) and the fact that $b_{i}>0$, we get

$$
\begin{aligned}
& \left\{1-\frac{1}{2}|r(\infty)|-\varepsilon\right\}\left(L_{h}^{n} \zeta^{n+1}, \zeta^{n+1}\right) \\
& \quad \leqslant \frac{1}{2}|r(\infty)|\left(L_{h}^{n} \zeta^{n}, \zeta^{n}\right)+c(\varepsilon k)^{-1}\left\{\left\|\zeta^{n}\right\|^{2}-\left\|\zeta^{n+1}\right\|^{2}\right\} .
\end{aligned}
$$

Since $|r(\infty)|<1$, we can choose $\varepsilon>0$ small enough so that for positive constants $c_{1}, c_{2}, c_{3}$ with $c_{3}>c_{2}+c_{1}$ we have

$$
\begin{aligned}
& \left\|\zeta^{n+1}\right\|^{2}+c_{3} k\left(L_{h}^{n} \zeta^{n+1}, \zeta^{n+1}\right) \\
& \quad \leqslant\left\|\zeta^{n}\right\|^{2}+\left(c_{2}+c_{1}\right) k\left(L_{h}^{n} \zeta^{n}, \zeta^{n}\right)-c_{1} k\left(L_{h}^{n} \zeta^{n}, \zeta^{n}\right) .
\end{aligned}
$$

Using inequalities (2.3) and $\left(L_{h}^{n+1} \zeta^{n+1}, \zeta^{n+1}\right) \leqslant(1+c k)\left(L_{h}^{n} \zeta^{n+1}, \zeta^{n+1}\right),(4.16)$ follows from (4.20).

Remark 4.1. It follows from (4.9) or (4.16) that $\left\|\left|U_{h}^{n}\right|\right\|\left\|_{c^{*}, n} \leqslant(1+\tilde{c} k)^{n}\right\| \mid U_{h}^{0}\|\|_{c^{*}, 0}$. Now if $\tilde{c}$ is negative, this could be interpreted as decay. For "long time" calculations, the dependence on $t^{*}$ of the constants in (2.4) and (2.3) plays an important role; if these constants grow with $t^{*}$, then $k$ must be chosen accordingly small in order to have $\tilde{c}<0$.

We have assumed above that $u^{0}$ is sufficiently smooth and compatible so that $u(t)$ is smooth for $0 \leqslant t \leqslant t^{*}$. Now since some of the constants $\tau_{i}$ may be greater than 1 , we shall henceforth assume that $u(t)$ is smooth for $0 \leqslant t \leqslant t^{* *}=t^{*}+$ $k \max \left\{0, \max _{i}\left(\tau_{i}-1\right)\right\}$.

THEOREM 4.3. Suppose that the eigenvalues of $A$ satisfy $\operatorname{Re} \lambda \geqslant 0, \lambda \neq 0$, and that (3.8) and (3.9) hold. Suppose $u^{0}$ is sufficiently smooth and compatible so that $u_{t}(t) \in H^{r} \cap H_{0}^{1}, u^{(\sigma)}(t) \in H^{2} \cap H_{0}^{1}, u^{(\sigma+1)}(t) \in L^{2}, 0 \leqslant t \leqslant t^{* *}$, and that a constant $c_{\mu}$ exists such that

$$
\left\|u^{(j)}(t)\right\|_{m} \leqslant c_{\mu}\left\|u^{0}\right\|_{\mu}, \quad m, j \geqslant 0,2 j+m \leqslant \mu, 0 \leqslant t \leqslant t^{* *},
$$

where $\mu=\max \{r+2,2(\sigma+1)\}$ and $\sigma=\min \{p, \nu\}$. 
Then, for any $c^{*}>0$, there exists $c=c\left(c^{*}\right)$ such that

$$
\left\|\mathscr{R}_{h}^{n} w^{n}-w^{n+1}\right\|_{c^{*}, n+1} \leqslant c k\left\{k^{\sigma}+k^{\sigma-1} h^{2}+k^{\sigma-1 / 2} h+h^{r}\right\}\left\|u^{0}\right\|_{\mu} .
$$

Proof. With $\tilde{W}^{n}=\left(w^{n, 1}, \ldots, w^{n, q}\right)^{T}, w^{n, j}=w\left(t^{n, j}\right)$, and $\tilde{F}^{n} \in \mathscr{S}_{h}$ given by

$$
\left(I+k A \tilde{\mathscr{L}}_{h}^{n}\right) \tilde{F}^{n}=-\left\{\frac{\Upsilon^{\sigma}}{\sigma}-A \Upsilon^{\sigma-1}\right\} e \frac{k^{\sigma}}{(\sigma-1) !} w^{(\sigma)}\left(t^{n}\right),
$$

we write for some $\tilde{E}^{n}$ in $\mathscr{S}_{h}$,

$$
\left(I+k A \tilde{\mathscr{L}}_{h}^{n}\right)\left(\tilde{W}^{n}+\tilde{F}^{n}+\tilde{E}^{n}\right)=e w^{n} .
$$

We let $\mathscr{P}_{0}, \tilde{\mathscr{P}}_{E}^{n}, \tilde{\mathscr{L}}^{n}$ denote the diagonal operators with entries $P_{0}, P_{E}^{n, i}=$ $T_{h}\left(t^{n, i}\right) L\left(t^{n, i}\right)$ and $L\left(t^{n, i}\right), i=1, \ldots, q$, respectively. With $\tilde{U}^{n}=\left(u^{n, 1}, \ldots, u^{n, q}\right)^{T}$ and using $\tilde{\mathscr{L}}_{h}^{n} \tilde{W}^{n}=\mathscr{P}_{0} \tilde{\mathscr{L}}^{n} \tilde{U}^{n}={ }_{\mathscr{P}_{0}} \tilde{U}_{t}^{n}$, from (4.24) we get

$$
\begin{aligned}
(I+ & \left.k A \tilde{\mathscr{L}}_{h}^{n}\right) \tilde{E}^{n}=-\left(I+k A \tilde{\mathscr{L}}_{h}^{n}\right) \tilde{F}^{n}+e w^{n}-\tilde{W}^{n}+k A \mathscr{P}_{0} \tilde{U}_{t}^{n} \\
& =-\left(I+k A \tilde{\mathscr{L}}_{h}^{n}\right) \tilde{F}^{n}+\left\{e w^{n}-\tilde{W}^{n}+k A \tilde{W}_{t}^{n}\right\}+k A\left(\mathscr{P}_{0} \tilde{U}_{t}^{n}-\tilde{W}_{t}^{n}\right) .
\end{aligned}
$$

With $z(t)=w(t)-P_{0} u(t)$, and $\tilde{\mathscr{D}}$ denoting the diagonal matrix satisfying $\tilde{\mathscr{D}} e=$ $\left\{\Upsilon^{\sigma} / \sigma-A \Upsilon^{\sigma-1}\right\} e$, from (3.9) we get

$$
\tilde{W}^{n}-e w^{n}-k A \tilde{W}_{t}^{n}=\frac{k^{\sigma}}{(\sigma-1) !} \tilde{\mathscr{D}} e w^{(\sigma)}\left(t^{n}\right)+\tilde{Z}_{1}^{n},
$$

where for $i=1, \ldots, q$, and assuming for simplicity that $\sigma \geqslant 2$,

$$
\begin{aligned}
\left(\tilde{Z}_{1}^{n}\right)_{i}= & \frac{-k^{\sigma}}{(\sigma-1) !} \tilde{D} e z^{(\sigma)}\left(t^{n}\right)+\frac{1}{(\sigma-1) !} \int_{t^{n}}^{t^{n, i}}\left(t^{n, i}-s\right)^{\sigma-1} z^{(\sigma)}(s) d s \\
& -\frac{k}{(\sigma-2) !} \sum_{j=1}^{q} a_{i j} \int_{t^{n}}^{t^{n, j}}\left(t^{n, j}-s\right)^{\sigma-2} z^{(\sigma)}(s) d s \\
& +\frac{1}{\sigma !} \int_{t^{n}}^{t^{n, i}}\left(t^{n, i}-s\right)^{\sigma} P_{0} u^{(\sigma+1)}(s) d s \\
& -\frac{k}{(\sigma-1) !} \sum_{j=1}^{q} a_{i j} \int_{t^{n}}^{t^{n, j}}\left(t^{n, j}-s\right)^{\sigma-1} P_{0} u^{(\sigma+1)}(s) d s .
\end{aligned}
$$

U'sing this and (4.23) in (4.25), we get

$$
\tilde{E}^{n}=-\left(I+k A \tilde{\mathscr{S}}_{h}^{n}\right)^{-1} \tilde{Z}_{1}^{n}+\left(I+k A \tilde{\mathscr{L}}_{h}^{n}\right)^{-1} k A\left(\mathscr{P}_{0} \tilde{U}_{t}^{n}-\tilde{W}_{t}^{n}\right) .
$$

Now, using the identity

(4.28) $k A \tilde{\mathscr{L}}_{h}^{n}\left(I+k A \tilde{\mathscr{L}}_{h}^{n}\right)^{-1}=I-\left(I+k A \tilde{\mathscr{L}}_{h}^{n}\right)^{-1}=\left(I+k A \tilde{\mathscr{L}}_{h}^{n}\right)^{-1} k A \tilde{\mathscr{L}}_{h}^{n}$,

from (4.24) we get

$$
\mathscr{R}_{h}^{n} w^{n}-w^{n+1}=w^{n}-w^{n+1}+b^{T} A^{-1}\left(\tilde{W}^{n}-e w^{n}\right)+b^{T} A^{-1} \tilde{F}^{n}+b^{T} A^{-1} \tilde{E}^{n} .
$$

Expanding $\tilde{W}^{n}$ in a Taylor series at $t=t^{n}$,

$$
b^{T} A^{-1}\left(\tilde{W}^{n}-e w^{n}\right)=\sum_{l=0}^{\sigma-1} b^{T} A^{-1} \Upsilon^{l+1} e \frac{k^{l+1}}{(l+1) !} w^{(l+1)}\left(t^{n}\right)+b^{T} A^{-1} \tilde{Z}_{2}^{n}
$$


where for $i=1, \ldots, q$,

$$
\begin{aligned}
\left(\tilde{Z}_{2}^{n}\right)_{i}=\frac{1}{\sigma !}\left\{-\left(k \tau_{i}\right)^{\sigma} \eta^{(\sigma)}\left(t^{n}\right)+\right. & \sigma \int_{t^{n}}^{t^{n . i}}\left(t^{n, i}-s\right)^{\sigma-1} \eta^{(\sigma)}(s) d s \\
& \left.+\int_{t^{n}}^{t^{n . i}}\left(t^{n, i}-s\right)^{\sigma} u^{(\sigma+1)}(s) d s\right\} .
\end{aligned}
$$

From (3.8) and (3.9) we have $b^{T} A^{-1} \Upsilon^{l+1} e=1$, for $0 \leqslant l \leqslant \min \{p-2, \nu-1\}$. Using this in (4.30), from (4.29) we get

$$
\begin{aligned}
\mathscr{R}_{h}^{n} w^{n}-w^{n+1}= & \sum_{l=0}^{\sigma-1} \frac{k^{l}}{l !} w^{(l)}\left(t^{n}\right)-w^{n+1}+b^{T} A^{-1}\left\{\Upsilon \sigma e \frac{k^{\sigma}}{\sigma !} w^{(\sigma)}\left(t^{n}\right)+\tilde{F}^{n}\right\} \\
& +b^{T} A^{-1} \tilde{E}^{n}+b^{T} A^{-1} \tilde{Z}_{2}^{n} .
\end{aligned}
$$

Using (4.28) and (4.23), we get

$$
\begin{aligned}
b^{T} A^{-1} & \left\{\Upsilon^{\sigma} \frac{k^{\sigma}}{\sigma !} e w^{(\sigma)}\left(t^{n}\right)+\tilde{F}^{n}\right\} \\
= & b^{T} \Upsilon^{\sigma-1} e \frac{k^{\sigma}}{(\sigma-1) !} w^{(\sigma)}\left(t^{n}\right)+b^{T} A^{-1}\left(I+k A \tilde{\mathscr{L}}_{h}^{n}\right)^{-1} \\
& \times k A \tilde{\mathscr{L}}_{h}^{n} \tilde{\mathscr{D}} e \frac{k^{\sigma}}{(\sigma-1) !} w^{(\sigma)}\left(t^{n}\right) \\
& \equiv \tilde{M}_{1}^{n}+\tilde{M}_{2}^{n} .
\end{aligned}
$$

Note that from (3.8), $b^{T} \Upsilon^{\sigma-1} e=1 / \sigma$. Hence, $\tilde{M}_{1}^{n}=\left(k^{\sigma} / \sigma !\right) w^{(\sigma)}\left(t^{n}\right)$. Using this and (4.32) in (4.31), we get

$$
\mathscr{R}_{h}^{n} w^{n}-w^{n+1}=\left(\sum_{l=0}^{0} \frac{k^{l}}{l !} w^{(l)}\left(t^{n}\right)-w^{n+1}\right)+\tilde{M}_{2}^{n}+b^{T} A^{-1} \tilde{E}^{n}+b^{T} A^{-1} \tilde{Z}_{2}^{n} .
$$

We now estimate the terms in (4.33). From (4.27), and using (4.4),

(4.34) $\left\|b^{T} A^{-1} \tilde{E}^{n}\right\|+\left\|\left(k L_{h}^{n}\right)^{1 / 2} b^{T} A^{-1} \tilde{E}^{n}\right\| \leqslant c\left\{\left\|\tilde{Z}_{1}^{n}\right\|+k\left\|\mathscr{P}_{0} \tilde{U}_{t}^{n}-\tilde{W}_{t}^{n}\right\|\right\}$.

Now from (2.1) and (2.7),

$$
\begin{aligned}
&\left\|\mathscr{P}_{0} \tilde{U}_{t}^{n}-\tilde{W}_{t}^{n}\right\| \leqslant c \max _{i}\left\|P_{0} u_{t}^{n, i}-w_{t}^{n, i}\right\| \\
& \leqslant \max _{i}\left\{\left\|P_{0} u_{t}^{n, i}-u_{t}^{n, i}\right\|+\left\|\eta_{t}^{n, i}\right\|\right\} \leqslant c h^{r}\left\|u^{0}\right\|_{r+2}, \\
&\left\|\tilde{Z}_{1}^{n}\right\| \leqslant c\left\{k^{\sigma} h^{2}+k^{\sigma+1}\right\}\left\|u^{0}\right\|_{2(\sigma+1)} .
\end{aligned}
$$

We have, by using $\tilde{\mathscr{L}}_{h}^{n} \tilde{\mathscr{D}}_{E}^{n}=\mathscr{\mathscr { D }}_{0} \tilde{\mathscr{L}}^{n}$ and (4.28),

$$
\begin{aligned}
\tilde{M}_{2}^{n}=\frac{k^{\sigma}}{(\sigma-1) !} b^{T} A^{-1}\left\{\left[I-\left(I+k A \tilde{\mathscr{L}}_{h}^{n}\right)^{-1}\right] \tilde{\mathscr{D}}\left[e w^{(\sigma)}\left(t^{n}\right)-\tilde{\mathscr{P}}_{E}^{n} e u^{(\sigma)}\left(t^{n}\right)\right]\right. \\
\left.+k\left(I+k A \tilde{\mathscr{L}}_{h}^{n}\right)^{-1} A \tilde{\mathscr{D}}_{0} \tilde{\mathscr{L}}^{n} e u^{(\sigma)}\left(t^{n}\right)\right\} .
\end{aligned}
$$

Using $\left\|\tilde{\mathscr{L}}^{n} e u^{(\sigma)}\left(t^{n}\right)\right\| \leqslant c\left\|u^{0}\right\|_{2(\sigma+1)}$ and (4.4) in (4.37), we get

$$
\left\|\tilde{M}_{2}^{n}\right\|+\left\|\left(k L_{h}^{n}\right)^{1 / 2} \tilde{M}_{2}^{n}\right\|
$$

$$
\begin{aligned}
\leqslant c k^{\sigma+1}\left\|u^{0}\right\|_{2(\sigma+1)}+c k^{\sigma} \max _{i}\{ & \left\|w^{(\sigma)}\left(t^{n}\right)-P_{E}^{n, i} u^{(\sigma)}\left(t^{n}\right)\right\| \\
& \left.+\left\|\left(k L_{h}^{n}\right)^{1 / 2}\left[w^{(\sigma)}\left(t^{n}\right)-P_{E}^{n, i} u^{(\sigma)}\left(t^{n}\right)\right]\right\|\right\} .
\end{aligned}
$$


Now from

$$
w^{(\sigma)}\left(t^{n}\right)-P_{E}^{n, i} u^{(\sigma)}\left(t^{n}\right)=w^{(\sigma)}\left(t^{n}\right)-u^{(\sigma)}\left(t^{n}\right)+\left(T^{n, i}-T_{h}^{n, i}\right) L^{n, i} u^{(\sigma)}\left(t^{n}\right),
$$

and using (2.2) and (2.7), we get

$$
\left\|w^{(\sigma)}\left(t^{n}\right)-P_{E}^{n, i} u^{(\sigma)}\left(t^{n}\right)\right\| \leqslant c h^{2}\left\|u^{0}\right\|_{2(\sigma+1)} .
$$

Similarly, from (2.2), (2.4) and (2.7),

$$
\begin{aligned}
\left\|\left(k L_{h}^{n}\right)^{1 / 2}\left[w^{(\sigma)}\left(t^{n}\right)-P_{E}^{n, i} u^{(\sigma)}\left(t^{n}\right)\right]\right\| \\
\leqslant c k^{1 / 2}\left\{\left\|w^{(\sigma)}\left(t^{n}\right)-u^{(\sigma)}\left(t^{n}\right)\right\|_{I}+\left\|\left(T^{n, i}-T_{h}^{n, i}\right) L^{n, i} u^{(\sigma)}\left(t^{n}\right)\right\|_{I}\right\} \\
\quad \leqslant c k^{1 / 2} h\left\|u^{0}\right\|_{2(\sigma+1)} .
\end{aligned}
$$

Using (4.39) and (4.40) in (4.38), we get

$$
\left\|\tilde{M}_{2}^{n}\right\|+\left\|\left(k L_{h}^{n}\right)^{1 / 2} \tilde{M}_{2}^{n}\right\| \leqslant c\left\{k^{\sigma+1}+k^{\sigma+1 / 2} h+k^{\sigma} h^{2}\right\}\left\|u^{0}\right\|_{2(\sigma+1)} .
$$

It remains to estimate the first and last terms in (4.33). These are similar, so only the first term will be considered. We have

$$
\begin{aligned}
\rho \equiv \sum_{l=0}^{\sigma} \frac{k^{l}}{l !} w^{(l)}\left(t^{n}\right)-w^{n+1} & \\
= & \frac{1}{\sigma !}\left\{k^{\sigma} \eta^{(\sigma)}\left(t^{n}\right)-\sigma \int_{t^{n}}^{t^{n+1}}\left(t^{n+1}-s\right)^{\sigma-1} \eta^{(\sigma)}(s) d s\right. \\
& \left.\quad-\int_{t^{n}}^{t^{n+1}}\left(t^{n+1}-s\right)^{\sigma} u^{(\sigma+1)}(s) d s\right\} .
\end{aligned}
$$

Now from (2.7) it follows that

$$
\|\rho\| \leqslant c k\left\{k^{\sigma-1} h^{2}+k^{\sigma}\right\}\left\|u^{0}\right\|_{2(\sigma+1)} .
$$

To estimate $\left\|\left(k L_{h}^{n}\right)^{1 / 2} \rho\right\|$, we write with $P_{E}^{n}=T_{h}^{n} L^{n}$,

$$
\begin{aligned}
\rho=\frac{1}{\sigma !} & \left\{k^{\sigma} \eta^{(\sigma)}\left(t^{n}\right)-\sigma \int_{t^{n}}^{t^{n+1}}\left(t^{n+1}-s\right)^{\sigma-1} \eta^{(\sigma)}(s) d s\right. \\
& \left.\quad+k^{\sigma}\left[I-P_{E}^{n}\right] u^{(\sigma)}\left(t^{n}\right)-\sigma \int_{t^{n}}^{t^{n+1}}\left(t^{n+1}-s\right)^{\sigma-1}\left[I-P_{E}^{n}\right] u^{(\sigma)}(s) d s\right\} \\
& +\frac{1}{\sigma !}\left\{k^{\sigma} P_{E}^{n} u^{(\sigma)}\left(t^{n}\right)-\sigma \int_{t^{n}}^{t^{n+1}}\left(t^{n+1}-s\right)^{\sigma-1} P_{E}^{n} u^{(\sigma)}(s) d s\right\} \\
\equiv & \rho_{1}+\rho_{2} .
\end{aligned}
$$

From (2.3), (2.4) and the Cauchy-Schwarz inequality,

$$
\begin{aligned}
\left(L_{h}^{n} \rho, \rho\right) & \leqslant c\|\rho\|_{I}^{2} \leqslant c\left\|\rho_{1}\right\|_{I}^{2}+c\left\|\rho_{2}\right\|_{I}^{2} \leqslant c\left\|\rho_{1}\right\|_{I}^{2}+c\left(L_{h}^{n} \rho_{2}, \rho_{2}\right) \\
& \leqslant c\left\|\rho_{1}\right\|_{I}^{2}+c\left\|L_{h}^{n} \rho_{2}\right\|\left(\|\rho\|+\left\|\rho_{1}\right\|\right) .
\end{aligned}
$$

Now using (2.2) and (2.7) we get $\left\|\rho_{1}\right\|+h\left\|\rho_{1}\right\|_{I} \leqslant c k^{\circ} h^{2}\left\|u^{0}\right\|_{2(\sigma+1)}$. Also, using $L_{h}^{n} P_{t:}^{n}=P_{0} L^{n}$, we have $\left\|L_{h}^{n} \rho_{2}\right\| \leqslant c k^{\sigma}\left\|u^{0}\right\|_{2(\sigma+1)}$. Hence,

$$
\left\|\left(k L_{h}^{n}\right)^{1 / 2} \rho\right\| \leqslant c k\left\{k^{\sigma}+k^{\sigma-1 / 2} h\right\}\left\|u^{0}\right\|_{2(\sigma+1)} .
$$

(4.22) now follows from (4.33)-(4.36), (4.38), (4.41)-(4.44) and (2.9). 
Remark 4.2. Using Young's inequality, we get $k^{\sigma-1} h^{2}+k^{\sigma-1 / 2} h \leqslant c\left\{k^{\sigma}+h^{r}\right\}$ under the mild restriction $2 \sigma \geqslant r$. The above inequality also follows from $h^{2}<c k$, for any constant $c$. Alternatively, we could have obtained the bound $k\left\{k^{\sigma}+h^{r}\right\}$ directly at the price of imposing slightly higher regularity on $u^{0}$.

Henceforth, we shall let $\mathscr{E}=\mathscr{E}(k, h, \sigma, r)=k^{\sigma}+k^{\sigma-1} h^{2}+k^{\sigma-1 / 2} h+h^{r}$. We have

THEOREM 4.4. Let $\left\{U_{h}^{n}\right\}_{n=0}^{n^{*}}$ be given by (3.1)-(3.3). Then under the conditions of Theorem 4.1, or Theorem 4.2 and Theorem 4.3, there exists a constant $c=c\left(c^{*}\right)$ such that

$$
\begin{gathered}
\max _{0 \leqslant n \leqslant n^{*}}\left\|U_{h}^{n}-w^{n}\right\|_{c^{*}, n} \leqslant c \mathscr{E}\left\|u^{0}\right\|_{\mu}, \\
\max _{0 \leqslant n \leqslant n^{*}}\left\|U_{h}^{n}-u^{n}\right\| \leqslant c \mathscr{E}\left\|u^{0}\right\|_{\mu},
\end{gathered}
$$

where $\mu=\max \{r+2,2(\sigma+1)\}, \sigma=\min \{p, \nu\}$ and $c^{*}$ is as in Theorem 4.1 or Theorem 4.2.

Proof. From (4.22) and (4.9) or (4.16),

$$
\left\|U_{h}^{n+1}-w^{n+1}\right\|_{c^{*}, n+1}^{2} \leqslant(1+\tilde{c} k)\left\|U_{h}^{n}-w^{n}\right\|_{c^{*}, n}^{2}+c k \mathscr{E}^{2}\left\|u^{0}\right\|_{\mu}^{2} \text {, }
$$

$$
0 \leqslant n \leqslant n^{*}-1
$$

A standard technique then yields

$$
\left\|U_{h}^{n}-w^{n}\right\|_{c^{*}, n}^{2} \leqslant(1+\tilde{c} k)^{n}\left\|U_{h}^{0}-w^{0}\right\|_{c^{*}, 0}^{2}+c k \mathscr{E}^{2}\left\|u^{0}\right\|_{\mu}^{2} \sum_{l=0}^{n-1}(1+\tilde{c} k)^{l} .
$$

Note that

$$
\sum_{l=0}^{n-1}(1+\tilde{c} k)^{l}= \begin{cases}n, & \tilde{c}=0, \\ (|\tilde{c}| k)^{-1}, & \tilde{c}<0 .\end{cases}
$$

It remains thus to estimate $\left\|U_{h}^{0}-w^{0}\right\|_{c^{*}, 0}$. But from (3.3), $\left(I+k \bar{\lambda} L_{h}^{0}\right)\left(U_{h}^{0}-w^{0}\right)$ $=P_{0} u^{0}-w^{0}$. Since $\bar{\lambda}>0,(2.1),(2.7)$, and (4.3) give at once $\left\|U_{h}^{0}-w^{0}\right\| \|_{c^{*}, 0} \leqslant$ $c^{r}\left\|u^{0}\right\|_{r}$.

Remark 4.3. The dependence on $t^{*}$ of the constant $c$ in (4.45) and (4.46) can be described in terms of the dependence on $t^{*}$ of the constants in (2.2)-(2.9) and $c_{\mu}$ in (4.21), and the sign of $\tilde{c}$. If $\tilde{c}>0$, then $c$ may depend on $t^{*}$ exponentially, regardless of other factors. If, on the other hand, $\tilde{c}<0$ and the above constants are independent of $t^{*}$, then $c$ is also independent of $t^{*}$.

5. Preconditioned Iterative Methods. The base scheme (3.1)-(3.3) involves solving at each time step a linear system of equations with coefficient matrix $I+k A \tilde{\mathscr{L}}_{h}^{n}$ (with respect to some basis for $S_{h}$ ). Two major difficulties are immediately revealed. Since $A$ is in general a full matrix (e.g., this is the case if $\nu=p), I+k A \tilde{\mathscr{L}}_{h}^{n}$ is, in general, a full $q$ by $q$ block matrix. The second difficulty stems from the fact that $\tilde{\mathscr{L}}_{h}^{n}$ depends on $n$, and hence requires new factorizations at each time step.

In this section, we shall describe two variants of the base scheme, which will substantially alleviate these difficulties, and at the same time, will preserve the stability and accuracy of the base scheme. Furthermore, we shall show that if the eigenvalues of $A$ are real, positive, and distinct, then the $q$ intermediate stages decouple, i.e., they can be solved independently and simultaneously. 
We begin by describing a class of Preconditioned Iterative Methods (or PIM) designed to avoid new matrix factorizations at each time step. Such iterative methods were used in the context of time stepping for parabolic problems by Douglas, Dupont and Ewing [13], and Bramble and Sammon [5].

Let $\mathscr{H}$ be a finite-dimensional Hilbert space with inner product $(\cdot, \cdot)_{\mathscr{H}}$ and norm $\|\cdot\|_{\mathscr{H}}=(\cdot, \cdot)_{\mathscr{H}}^{1 / 2}$. Suppose $M, M_{0}$ are symmetric positive definite operators on $\mathscr{H}$, and are equivalent in the sense that for constants $c_{2} \geqslant c_{1}>0$,

$$
c_{1}\left(M_{0} \phi, \phi\right)_{\mathscr{H}} \leqslant(M \phi, \phi)_{\mathscr{H}} \leqslant c_{2}\left(M_{0} \phi, \phi\right)_{\mathscr{H}} \quad \forall \phi \in \mathscr{H} .
$$

Suppose also that we want to solve the linear system $M x=b, b \in \mathscr{H}$, and that solving the linear system $M_{0} y=z$ is relatively inexpensive. Given $x^{(0)} \in \mathscr{H}$, these PIMs (with $M_{0}$ as the preconditioner) generate approximations $\left\{x^{(j)}\right\}_{j=0}^{J}$ to $x$. These iterative methods possess the following properties (cf. [1], [5]).

(1) Given $\left\{x^{(j)}\right\}_{j=0}^{J}$, calculating $x^{(J+1)}$ involves only evaluating $M \phi, M_{0} \phi$, $\phi \in \mathscr{H}$, computing inner products $(M \phi, \phi)_{\mathscr{H}},\left(M_{0} \phi, \phi\right)_{\mathscr{H}}$ and solving linear systems $M_{0} \phi=\psi, \psi \in \mathscr{H}$.

(2) There is a smooth decreasing function $\rho:(0,1] \rightarrow[0,1)$ with $\rho(1)=0$, and such that (with $c_{1}, c_{2}$ as in (5.1)),

$$
\left\|M_{0}^{1 / 2}\left(x^{(j)}-x\right)\right\|_{\mathscr{H}} \leqslant c\left[\rho\left(c_{1} / c_{2}\right)\right]^{j}\left\|M_{0}^{1 / 2}\left(x^{(0)}-x\right)\right\|_{\mathscr{H}} .
$$

A particularly efficient PIM is the preconditioned conjugate-gradient method, with $\rho(s)=(1-\sqrt{s}) /(1+\sqrt{s})$. Also, it does not require knowledge of the constants $c_{1}$ and $c_{2}$.

In the sequel, we shall let $\mathscr{H}=S_{h}$ and equip it with the $L^{2}$-norm.

Since $\tilde{\mathscr{L}}_{h}^{n}$ contains operators $L_{h}(\cdot)$ evaluated at different time levels, we shall consider the splitting $I+k A \mathscr{L}_{h}^{n}-k A\left(\mathscr{L}_{h}^{n}-\tilde{\mathscr{L}}_{h}^{n}\right)$ and use it to define the iteration

$$
\left(I+k A \mathscr{L}_{h}^{n}\right) V_{l+1}^{n}=e U_{h}^{n}+k A\left(\mathscr{L}_{h}^{n}-\tilde{\mathscr{L}}_{h}^{n}\right) V_{l}^{n}, l=0,1, \ldots, ; V_{0}^{n} \in \mathscr{S}_{h} .
$$

We shall refer to this as the "outer iteration". The following result concerns the application of (5.3) to the system

$$
\left(I+k A \tilde{\mathscr{L}}_{h}^{n}\right) V^{n}=e U_{h}^{n} .
$$

Proposition 5.1. Let $V^{n} \in \mathscr{S}_{h}$ be the solution of (5.4) and let $\left\{V_{l}^{n}\right\}_{l \geqslant 0}$ be given by (5.3). Then for any $c^{*}>0$,

$$
\left\|V^{n}-V_{l+1}^{n}\right\|_{c^{*}, 0} \leqslant c\left(c^{*}\right) k\left\|V^{n}-V_{l}^{n}\right\|_{c^{*}, 0}, \quad l \geqslant 0 .
$$

Proof. From $\left(I+k A \mathscr{L}_{h}^{n}\right)\left(V^{n}-V_{l+1}^{n}\right)=k A\left(\mathscr{L}_{h}^{n}-\tilde{\mathscr{L}}_{h}^{n}\right)\left(V^{n}-V_{l}^{n}\right)$, written as

$$
\begin{aligned}
V^{n}-V_{l+1}^{n}= & \left(I+k A \mathscr{L}_{h}^{n}\right)^{-1}\left(k \mathscr{L}_{h}^{n}\right)^{1 / 2} A\left(\mathscr{T}_{h}^{n}\right)^{1 / 2}\left(\mathscr{L}_{h}^{n}-\tilde{\mathscr{L}}_{h}^{n}\right)\left(\mathscr{T}_{h}^{n}\right)^{1 / 2} \\
& \times\left(k \mathscr{L}_{h}^{n}\right)^{1 / 2}\left(V^{n}-V_{l}^{n}\right),
\end{aligned}
$$

(5.5) follows by using (2.5), (4.3) and (2.9).

In what follows, we shall be able to restrict the number of outer iterations to one for $n \geqslant \sigma-1$. For $n<\sigma-1$, we need not iterate more than $\sigma$ times.

We shall approximate $V_{l+1}^{n}$ in (5.3) using a PIM, which shall be referred to as the "inner iteration". Let $S^{-1} \Lambda S$ be the Jordan decomposition of $A$, with $\Lambda$ lower triangular. With $V_{l}^{n, j_{l}}$ an approximation to $V^{n}$, we write (5.3) in the more convenient 
form (redefining $V_{l+1}^{n}$ )

$$
\begin{aligned}
& \left(I+k \Lambda \mathscr{L}_{h}^{n}\right) S V_{l+1}^{n}=S e U_{h}^{n}+k \Lambda S\left(\mathscr{L}_{h}^{n}-\tilde{\mathscr{L}}_{h}^{n}\right) V_{l}^{n, j_{l}}, \quad l=0,1, \ldots,
\end{aligned}
$$

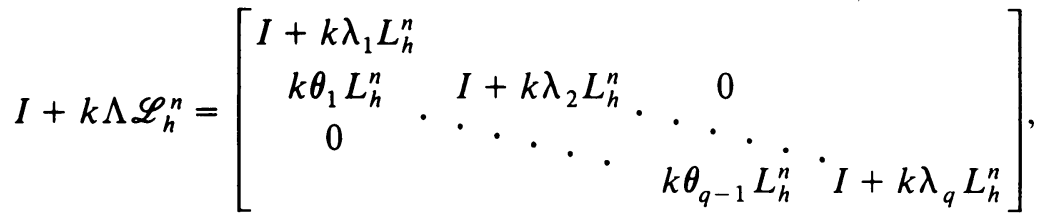

where for $q \geqslant 1, \theta_{i}=0$ or 1 . With $\theta_{0}=0$, we write (5.6) as

$$
\begin{aligned}
\left(I+k \lambda_{i} L_{h}^{n}\right)\left(S V_{l+1}^{n}\right)_{i}= & \left(S e U_{h}^{n}+k \Lambda S\left(\mathscr{L}_{h}^{n}-\tilde{\mathscr{L}}_{h}^{n}\right) V_{l}^{n, j_{l}}\right)_{i} \\
& -k \theta_{i-1} L_{h}^{n}\left(S V_{l+1}^{n}\right)_{i-1}, \quad i=1, \ldots, q .
\end{aligned}
$$

For $\bar{\lambda}>0$ fixed, $I+k \bar{\lambda} L_{h}^{0}$ and $I+k \lambda_{i} L_{h}^{n}$ are symmetric, positive definite and equivalent in the sense of (5.1). With an initial guess $V_{l+1}^{n, 0}$, we apply $j_{l+1}$ iterations of the PIM recursively for each $i, i=1, \ldots, q$. We obtain

$$
\left\|V_{l+1}^{n}-V_{l+1}^{n, j_{l+1}}\right\|_{\lambda, 0} \leqslant \beta_{l+1}\left\|V_{l+1}^{n}-V_{l+1}^{n, 0}\right\|_{\lambda, 0}, \quad \beta_{l+1}<1 .
$$

We now make the following simple but important observation: If the eigenvalues of $A$ are distinct, then $\theta_{i}=0, i=1, \ldots, q-1$, thus the matrix $I+k \Lambda L_{h}^{n}$ becomes diagonal and (5.7) decouples into $q$ independent linear systems, which can be handled simultaneously, or in "parallel", on a computer with at least $q$ independent processors. An equally important fact is that we know how to construct IRK methods having the required properties; specifically, the (real) IRKC methods described in Section 3. These methods are of arbitrarily high, optimal order (optimal in the sense that $\nu=p=q+1$ ) and stable in the sense of (3.6) (or (3.7) for $q=2$ ), and with eigenvalues of $A$ that are real, positive, and distinct. A reasonable choice for $\bar{\lambda}$ would then be $q^{-1} \sum_{j=1}^{q} \lambda_{i}$.

We next analyze a procedure that consists of a cycle of one outer iteration followed by a certain number of inner iterations.

Proposition 5.2. Suppose the eigenvalues of $A$ a real and positive. Let $V_{l}^{n_{1} j_{l}}$ be an approximation to $V^{n}$, the solution of (5.4), and let $V_{l+1}^{n, j_{l+1}}$ be an approximation to $V_{l+1}^{n}$, the solution of (5.6), obtained by applying $j_{l+1}$ iterations of the PIM with $I+k \bar{\lambda} L_{h}^{0}$ as preconditioner, $\bar{\lambda}>0$, and initial guess $V_{l+1}^{n, 0}=V_{l}^{n, j_{l}}$. Then

$$
\left\|V^{n}-V_{l+1}^{n, j_{l+1}}\right\|_{\lambda, 0} \leqslant\left\{c k+\beta_{l+1}\right\}\left\|V^{n}-V_{l}^{n, j_{l}}\right\|_{\lambda, 0} .
$$

Proof. From (5.5), (5.8) and since $V_{j_{l+1}}^{n .0}=V_{n}^{n, j_{l}}$,

$$
\begin{aligned}
\left\|V^{n}-V_{l+1}^{n, j_{l+1}}\right\|_{\lambda, 0} & \leqslant\left\|V^{n}-V_{l+1}^{n}\right\|_{\lambda_{, 0}}+\left\|V_{l+1}^{\prime \prime}-V_{l+1}^{n, j_{l+1}}\right\|_{\lambda_{, 0}} \\
& \leqslant c k\left\|V^{n}-V_{l}^{n, j_{l}}\right\|_{\lambda_{\lambda, 0}}+\beta_{l+1}\left\|V_{l+1}^{n}-V_{l}^{n, j_{l}}\right\|_{\lambda_{, 0} .}
\end{aligned}
$$

Also,

$$
\begin{aligned}
\left\|V_{l+1}^{n}-V_{l}^{n, j_{l}}\right\|_{\lambda, 0} & \leqslant\left\|V^{n}-V_{l+1}^{n}\right\|\left\|_{\lambda, 0}+\right\| V^{n}-V_{l}^{n, j_{l}} \|_{\lambda, 0} \\
& \leqslant(c k+1)\left\|V^{n}-V_{l}^{n, j_{l}}\right\|_{\lambda, 0} .
\end{aligned}
$$

Using this inequality in the previous one, we get (5.9). 
For simplicity, we shall take $\beta_{0}=\beta_{1}=\cdots \equiv \beta_{n}$. We are now in a position to define and analyze the first variant of the base scheme. Let $U_{h}^{0}$ be given by (3.3) and assume $\left\{U_{h}^{j}\right\}_{j=1}^{n}$ is given. We define the initial approximation $V_{0}^{n, 0}$ to $V^{n}$, the solution (5.4), by

$$
\left(V_{0}^{n, 0}\right)_{i}=\sum_{j=0}^{m} \gamma_{i j}^{m} U_{h}^{n-j}, \quad m=\min \{\sigma-1, n\}, i=1, \ldots, q,
$$

where the coefficients $\gamma_{i j}^{m}$ are given by

$$
\left\{\begin{array}{l}
\gamma_{i j}^{m}=(-1)^{j} \frac{\prod_{l=0 ; l \neq j}^{m}\left(l+\tau_{i}\right)}{j !(m-j) !}, \quad i=1, \ldots, q, j=0, \ldots, m, m \geqslant 1, \\
\gamma_{i 0}^{0}=1, \quad i=1, \ldots, q .
\end{array}\right.
$$

With $V_{0}^{n, 0}$ as initial guess, we obtain an approximation $V_{\sigma-m}^{n, j_{j}-m}$ to $V^{n}$ by applying the outer-inner cycle $\sigma-m$ times with $\beta_{n}=c k$ (note that $\sigma-m=1$ for $n \geqslant \sigma-1$ ). We then let (along $\left(3.1^{\prime}\right)$ )

$$
U_{h}^{n+1}=\left(1-b^{T} A^{-1} e\right) U_{h}^{n}+b^{T} A^{-1} V_{o-m}^{n-j_{c}-m} .
$$

We have

THEOREM 5.1. Suppose the conditions of Theorem 4.3 are satisfied, (3.4) holds and that the eigenvalues of $A$ are real positive. Let the sequence $\left\{U_{h}^{n}\right\}_{n=0}^{n^{*}}$ of fully discrete approximations be generated by the first variant of the base scheme. Then, for some constant $c>0$,

$$
\begin{gathered}
\max _{0 \leqslant n \leqslant n^{*}}\left\|U_{h}^{n}-w^{n}\right\|_{c^{*}, n} \leqslant c \mathscr{E}\left\|u^{0}\right\|_{\mu}, \\
\max _{0 \leqslant n \leqslant n^{*}}\left\|U_{h}^{n}-u_{h}^{n}\right\| \leqslant c \mathscr{E}\left\|u^{0}\right\|_{\mu},
\end{gathered}
$$

where $c^{*}$ is as in Theorem 4.1 and $\mathscr{E}$ as in Theorem 4.4.

Proof. With $\zeta^{j}=w^{j}-U_{h}^{j}$, we consider the error equation

$$
\zeta^{n+1}=w^{n+1}-\mathscr{R}_{h}^{n} w^{n}+\mathscr{R}_{h}^{n} \zeta^{n}+\mathscr{R}_{h}^{n} U_{h}^{n}-U_{h}^{n+1} .
$$

Now since $\beta_{n}=c k$,

$$
\begin{aligned}
\left\|\mathscr{R}_{h}^{n} U_{h}^{n}-U_{h}^{n+1}\right\|_{c^{*}, n+1} & =\left\|b^{T} A^{-1}\left(V^{n}-V_{\sigma-m}^{n, j_{o}-m}\right)\right\|_{c^{*}, n+1} \\
& \leqslant c k^{\sigma-m}\left\|V^{n}-V_{0}^{n, 0}\right\|_{c^{*}, n+1},
\end{aligned}
$$

from (5.9), (5.12) and (2.9). Now with $\tilde{W}^{n}, \tilde{F}^{n}, \tilde{E}^{n}$ as in the proof of Theorem 4.3, letting $\Gamma_{j}^{m}=\operatorname{diag}\left\{\gamma_{1 j}^{m}, \gamma_{2 j}^{m}, \ldots, \gamma_{q j}^{m}\right\}$,

$$
\begin{aligned}
V^{n}- & V_{0}^{n, 0}=V^{n}-\sum_{j=0}^{m} \Gamma_{j}^{m} e U_{h}^{n-j} \\
= & \sum_{j=0}^{m} \Gamma_{j}^{m} e \zeta^{n-j}-\left(I+k A \tilde{\mathscr{L}}_{h}^{n}\right)^{-1} e \zeta^{n}+\tilde{F}^{n}+\tilde{E}^{n}+\left(\tilde{W}^{n}-\sum_{j=0}^{m} \Gamma_{j}^{m} e w^{n-j}\right) .
\end{aligned}
$$

From (2.7) and (5.11) it follows that

$$
\text { (5.17) }\left\|\tilde{W}^{n}-\sum_{j=0}^{m} \Gamma_{j}^{m} e w^{n-j}\right\|_{c^{*}, n+1} \leqslant c k^{m+1}\left\|u^{0}\right\|_{2(m+2)}, \quad m=0,1, \ldots, \sigma-1 .
$$


Now as in the proof of Theorem 4.3, it can be shown that

$$
\left\|\tilde{F}^{n}+\tilde{E}^{n}\right\|_{c^{*}, n+1} \leqslant c\left\{k^{\sigma}+k h^{r}\right\}\left\|u^{0}\right\|_{\mu} .
$$

Using (4.4), (5.17), (5.18) and (2.9), we get

$$
\left\|V^{n}-V_{0}^{n, 0}\right\|_{c^{*}, n+1} \leqslant c \sum_{j=0}^{m}\left\|\zeta^{n-j}\right\|_{c^{*}, n-j}+c k\left\{k^{m}+h^{r}\right\}\left\|u^{0}\right\|_{\mu} .
$$

Using this and (5.16) in (5.15) together with (4.9) and (4.22), we get for $0 \leqslant n \leqslant n^{*}$,

$$
\left\|\zeta^{n+1}\right\|_{c^{*}, n+1}^{2} \leqslant(1+c k)\left\|\zeta^{n}\right\|_{c^{*}, n}^{2}+c k \sum_{j=1}^{m}\left\|\zeta^{n-j}\right\|_{c^{*}, n-j}^{2}+c k \mathscr{E}^{2}\left\|u^{0}\right\|_{\mu}^{2} .
$$

(5.13), and then (5.14), follow at once.

In contrast to Theorem 4.4, the constant $c$ in (5.13) may depend (exponentially) on $t^{*}$ (cf. Remark 4.3). Also, following each outer iteration, the inner iterations must produce an $O(k)$ refinement, which in view of (5.2) will require $O\left(\ln k^{-1}\right)$ iterations per step. We shall next analyze a second variant of the base scheme which is superior in both respects. It is similar to Algorithm 2 of Bramble and Sammon [5]. This algorithm requires the IRK methods to be dissipative in the sense of (3.6) or as in Theorem 4.2, and necessitates the use of initial approximations more accurate than (5.10), (5.11). However, under these conditions, we will show that we need to take $\beta_{n}=c t_{n-\sigma}^{1 / 2}$, for some $0<c<1$, thus requiring (as in [5]) an average number of inner iterations per step that is independent of $k$. Also, the dependence on $t^{*}$ of the error constant is similar to that in Theorem 4.4.

For the second variant of the base scheme, with $U_{h}^{0}$ given by (3.3), we generate $U_{h}^{1}, \ldots, U_{h}^{\sigma+1}$ using the first variant. For $n \geqslant \sigma+2$, we shall need to use improved initial approximations to $V^{n}$. For technical reasons, we are unable to use $U_{h}^{n}, U_{h}^{n-1}, \ldots$ as before. We shall instead use the intermediate stages. Assuming $U_{h}^{0}$, $\left\{U_{h}^{l+1}, V_{\sigma-m}^{l, j_{\sigma-m}}\right\}_{l=0}^{n-1}, n \geqslant \sigma+1$, given, we obtain an initial approximation $V_{0}^{n, 0}$ by

$$
V_{0}^{n, 0}=\sum_{l=1}^{\sigma+1}(-1)^{l-1}\left(\begin{array}{c}
\sigma+1 \\
l
\end{array}\right) V_{\sigma-m}^{n-l, j_{\sigma-m}}, \quad n=\sigma+1, \ldots, n^{*}-1
$$

We then apply a single outer iteration followed by $j_{1}$ inner iterations to obtain

$$
\left\|V^{n}-V_{1}^{n, j}\right\|_{c^{*}, n+1} \leqslant\left\{c k+\beta_{n}\right\}\left\|V^{n}-V_{0}^{n, 0}\right\|_{c^{*}, n+1} .
$$

Our first task will be to estimate $\left\|V^{n}-V_{0}^{n, 0}\right\|_{c^{*}, n+1}$. Letting $G^{l}=V^{l}-V_{\sigma-m}^{l, j_{\sigma-m}}$, $l=0,1, \ldots, n^{*}-1$, we have

Proposition 5.3. Suppose the conditions of Theorem 4.3 and Proposition 5.2 are satisfied and let $V_{0}^{n, 0}$ be given by (5.19). Then for any $c^{*}>0$,

$$
\begin{aligned}
&\left\|V^{n}-V_{0}^{n, 0}\right\| \|_{c^{*}, n+1} \leqslant c \sum_{l=0}^{\sigma}\left\|\zeta^{n-l}-\zeta^{n-l-1}\right\|_{c^{*}, n-l}+c \sum_{l=1}^{\sigma+1}\left\|G^{n-l}\right\| \|_{c^{*}, n-l} \\
&+c k \sum_{l=1}^{\sigma+1}\left\|\zeta^{n-l}\right\|_{c^{*}, n-l}+c k \mathscr{E}\left\|u^{0}\right\|_{\mu}, \\
& n=\sigma+1, \ldots, n^{*}-1 .
\end{aligned}
$$


Proof. We have

$$
\begin{aligned}
V^{n}-V_{0}^{n, 0}=\left(I+k A \tilde{\mathscr{L}}_{h}^{n}\right)^{-1} e U_{h}^{n}-\sum_{l=1}^{\sigma+1}(-1)^{l-1}\left(\begin{array}{c}
\sigma+1 \\
l
\end{array}\right) V_{\sigma-m}^{n-l, j_{\sigma-m}} \\
=\sum_{l=0}^{\sigma+1}(-1)^{l}\left(\begin{array}{c}
\sigma+1 \\
l
\end{array}\right)\left(I+k A \tilde{\mathscr{L}}_{h}^{n-l}\right)^{-1} e U_{h}^{n-l}+\sum_{l=1}^{\sigma+1}(-1)^{l-1}\left(\begin{array}{c}
\sigma+1 \\
l
\end{array}\right) G^{n-l} .
\end{aligned}
$$

Now,

$$
\begin{aligned}
& \sum_{l=0}^{\sigma+1}(-1)^{l}\left(\begin{array}{c}
\sigma+1 \\
l
\end{array}\right)\left(I+k A \tilde{\mathscr{L}}_{h}^{n-l}\right)^{-1} e U_{h}^{n} h^{n-l} \\
&=\sum_{l=0}^{\sigma+1}(-1)^{l}\left(\begin{array}{c}
\sigma+1 \\
l
\end{array}\right)\left(I+k A \tilde{\mathscr{L}}_{h}^{n-l}\right)^{-1} e\left[w^{n-l}-\zeta^{n-l}\right] \\
&=-\left(I+k A \tilde{\mathscr{L}}_{h}^{n}\right)^{-1} e \sum_{l=0}^{\sigma}(-1)\left(\begin{array}{l}
\sigma \\
l
\end{array}\right)\left(\zeta^{n-l}-\zeta^{n-l-1}\right) \\
&-\sum_{l=1}^{\sigma+1}(-1)^{l}\left(\begin{array}{c}
\sigma+1 \\
l
\end{array}\right)\left\{\left(I+k A \tilde{\mathscr{L}}_{h}^{n-l}\right)^{-1}-\left(I+k A \tilde{\mathscr{L}}_{h}^{n}\right)^{-1}\right\} e \zeta^{n-l} \\
&+\sum_{l=0}^{\sigma+1}(-1)^{l}\left(\begin{array}{c}
\sigma+1 \\
l
\end{array}\right)\left(\tilde{F}^{n-l}+\tilde{E}^{n-l}+\tilde{W}^{n-l}\right)
\end{aligned}
$$

where $\tilde{F}^{n-1}, \tilde{E}^{n-1}, \tilde{W}^{n-1}$, are as in the proof of Theorem 4.3. Now using the identity

$$
\begin{aligned}
(I+ & \left.k A \tilde{\mathscr{L}}_{h}^{n-l}\right)^{-1}-\left(I+k A \tilde{\mathscr{L}}_{h}^{n}\right)^{-1} \\
& =\left(I+k A \tilde{\mathscr{L}}_{h}^{n-l}\right)^{-1} k A\left(\tilde{\mathscr{L}}_{h}^{n}-\tilde{\mathscr{L}}_{h}^{n-l}\right)\left(I+k A \tilde{\mathscr{L}}_{h}^{n}\right)^{-1},
\end{aligned}
$$

and using the techniques of Section 4 ,

$$
\begin{gathered}
\left\|\sum_{l=1}^{\sigma+1}(-1)^{l}\left(\begin{array}{c}
\sigma+1 \\
l
\end{array}\right)\left\{\left(I+k A \tilde{\mathscr{L}}_{h}^{n-l}\right)^{-1}-\left(I+k A \tilde{\mathscr{L}}_{h}^{n}\right)^{-1}\right\} e \zeta^{n-l}\right\|_{c^{*}, n+1} \\
\leqslant c k \sum_{l=1}^{\sigma+1}\left\|\zeta^{n-l}\right\|_{c^{*}, n-l}
\end{gathered}
$$

Also, as in the proof of Theorem 4.3,

$$
\left\|\sum_{l=0}^{\sigma+1}(-1)^{l}\left(\begin{array}{c}
\sigma+1 \\
l
\end{array}\right) \tilde{E}^{n-l}\right\|\left\|_{c^{*}, n+1} \leqslant c k \mathscr{E}\right\| u^{0} \|_{\mu} .
$$

Now from (4.23), with $\tilde{D}$ as in (4.26),

$$
\begin{aligned}
& \sum_{l=0}^{\sigma+1}(-1)^{l}\left(\begin{array}{c}
\sigma+1 \\
l
\end{array}\right) \tilde{F}^{n-l} \\
&=-\sum_{l=0}^{\sigma+1}(-1)^{l}\left(\begin{array}{c}
\sigma+1 \\
l
\end{array}\right)\left(I+k A \tilde{\mathscr{L}}_{h}^{n-l}\right)^{-1} \tilde{\mathscr{D}} e \frac{k^{\sigma}}{(\sigma-1) !} w^{(\sigma)}\left(t^{n-l}\right) \\
&=-\frac{k^{\sigma}}{(\sigma-1) !} \sum_{l=0}^{\sigma}(-1)^{l}\left(\begin{array}{c}
\sigma \\
l
\end{array}\right)\left(I+k A \tilde{\mathscr{L}}_{h}^{n}\right)^{-1} \tilde{\mathscr{D}}_{e}\left[w^{(\sigma)}\left(t^{n-l}\right)-w^{(\sigma)}\left(t^{n-l-1}\right)\right] \\
&-\frac{k^{\sigma}}{(\sigma-1) !} \sum_{l=1}^{\sigma+1}(-1)^{l}\left(\begin{array}{c}
\sigma+1 \\
l
\end{array}\right)\left\{\left(I+k A \tilde{\mathscr{L}}_{h}^{n-l}\right)^{-1}\right. \\
&\left.-\left(I+k A \tilde{\mathscr{L}}_{h}^{n}\right)^{-1}\right\} \tilde{\mathscr{D}} e w^{(\sigma)}\left(t^{n-l}\right)
\end{aligned}
$$


Hence, using (4.4), (5.23) and as in the proof of Theorem 4.3,

$$
\left\|\sum_{l=0}^{\sigma+1}(-1)^{l}\left(\begin{array}{c}
\sigma+1 \\
l
\end{array}\right) \tilde{F}^{n-l}\right\|\left\|_{c^{*}, n+1} \leqslant c k \mathscr{E}\right\| u^{0} \|_{\mu} .
$$

Finally, using the fact that $\sum_{l=0}^{\sigma+1}(-1)^{l}\left({ }^{\sigma+1} l\right) g\left(\alpha+t^{n-l}\right)=0$, where $\alpha$ is a fixed scalar and $g$ is any polynomial of degree $\leqslant \sigma$, and using techniques of Theorem 4.3 (in estimating $\rho$ ),

$$
\left\|\sum_{l=0}^{\sigma+1}(-1)^{l}\left(\begin{array}{c}
\sigma+1 \\
l
\end{array}\right) \tilde{W}^{n-l} \mid\right\|_{c^{*}, n+1} \leqslant c k \mathscr{E}\left\|u^{0}\right\|_{\mu} .
$$

Using (5.24)-(5.27) in (5.22), we get (5.21).

Let $\overline{\mathscr{R}}_{h}^{n}=\left(L_{h}^{n}\right)^{1 / 2} \mathscr{R}_{h}^{n}\left(T_{h}^{n}\right)^{1 / 2}, Q=Q\left(k L_{h}^{n}\right),(r(z)=P(z) / Q(z)), \zeta^{n}=w^{n}-U_{h}^{n}$, $n=0,1, \ldots, n^{*}-1$. We have:

Proposition 5.4. Suppose the eigenvalues of $A$ satisfy $\operatorname{Re} \lambda \geqslant 0, \lambda \neq 0$ and that (3.5) holds with $\delta>0$. Then there exists $c_{\delta}>0$ such that for any $c^{*}>0$,

$$
\begin{aligned}
& c_{\delta}\left\|\zeta^{n+1}-\zeta^{n}\right\|_{c^{*}, n+1}^{2}+\left(\left[I-\mathscr{R}_{h}^{n}\right] \zeta^{n+1}, \zeta^{n+1}\right) \\
& \quad+c^{*}\left(\left[I-\overline{\mathscr{R}}_{h}^{n}\right]\left(k L_{h}^{n}\right)^{1 / 2} \zeta^{n+1},\left(k L_{h}^{n}\right)^{1 / 2} \zeta^{n+1}\right) \\
& \leqslant \\
& \quad\left(\left[I-\mathscr{R}_{h}^{n-1}\right] \zeta^{n}, \zeta^{n}\right)+c^{*}\left(\left[I-\overline{\mathscr{R}}_{h}^{n-1}\right]\left(k L_{h}^{n-1}\right)^{1 / 2} \zeta^{n},\left(k L_{h}^{n-1}\right)^{1 / 2} \zeta^{n}\right) \\
& \quad+c k^{2}\left\|\zeta^{n+1}\right\|_{c^{*}, n+1}^{2} \\
& \quad+c\left\{\left\|\mathscr{R}_{h}^{n} w^{n}-w^{n+1}\right\|_{c^{*}, n+1}^{2}+\left\|\mathscr{R}_{h}^{n} U_{h}^{n}-U_{h}^{n+1}\right\|_{c^{*}, n+1}^{2}\right\} \\
& \quad+c k\left\{\left\|\left(k L_{h}^{n}\right)^{1 / 2} Q^{-1 / 2} \zeta^{n}\right\|^{2}+\left\|\left(k L_{h}^{n}\right)^{q / 2} Q^{-1 / 2} \zeta^{n}\right\|^{2}\right. \\
& \left.\quad+\left\|\left(k L_{h}^{n}\right)^{(q+1) / 2} Q^{-1 / 2} \zeta^{n}\right\|^{2}\right\}, \quad 1 \leqslant n \leqslant n^{*}-1 .
\end{aligned}
$$

Proof. From (5.15) we get

$$
\begin{aligned}
\left(\left[I+\mathscr{R}_{h}^{n}\right]\left[\zeta^{n+1}-\zeta^{n}\right], \zeta^{n+1}-\zeta^{n}\right)+\left(\left[I-\mathscr{R}_{h}^{n}\right] \zeta^{n+1}, \zeta^{n+1}\right) \\
=\left(\left[I-\mathscr{R}_{h}^{n-1}\right] \zeta^{n}, \zeta^{n}\right)-\left(\left[\mathscr{R}_{h}^{n}-\mathscr{R}_{h}^{n-1}\right] \zeta^{n}, \zeta^{n}\right)+\left(\mathscr{R}_{h}^{n} \zeta^{n}, \zeta^{n+1}\right) \\
\quad-\left(\mathscr{R}_{h}^{n} \zeta^{n+1}, \zeta^{n}\right)+2\left(\left[w^{n+1}-\mathscr{R}_{h}^{n} w^{n}\right]+\left[\mathscr{R}_{h}^{n} U_{h}^{n}-U_{h}^{n+1}\right], \zeta^{n+1}-\zeta^{n}\right) .
\end{aligned}
$$

Writing $I+\mathscr{R}_{h}^{n}=I+r_{h}^{n}+\left(\mathscr{R}_{h}^{n}-r_{h}^{n}\right)$, from (3.5) and (4.14) it follows that for some constant $c(\delta)>0$,

$$
\left(\left[I+\mathscr{R}_{h}^{n}\right]\left[\zeta^{n+1}-\zeta^{n}\right], \zeta^{n+1}-\zeta^{n}\right) \geqslant c(\delta)\left\|\zeta^{n+1}-\zeta^{n}\right\|^{2} .
$$

Also,

$$
\begin{gathered}
\mathscr{R}_{h}^{n}-\mathscr{R}_{h}^{n-1}=r_{h}^{n}-r_{h}^{n-1}+\left(\mathscr{R}_{h}^{n}-r_{h}^{n}\right)-\left(\mathscr{R}_{h}^{n-1}-r_{h}^{n-1}\right), \\
r_{h}^{n}-r_{h}^{n-1}=-k b^{T}\left(I+k A \mathscr{L}_{h}^{n}\right)^{-1}\left(\mathscr{L}_{h}^{n}-\mathscr{L}_{h}^{n-1}\right)\left(I+k A \mathscr{L}_{h}^{n-1}\right)^{-1} e .
\end{gathered}
$$

Hence it follows from (2.5), (2.6), (4.3), (4.13) and (4.14) that

$$
\left\|\left(T_{h}^{n}\right)^{(1-j) / 2}\left(\mathscr{R}_{h}^{n}-\mathscr{R}_{h}^{n-1}\right)\left(T_{h}^{n}\right)^{(1-i) / 2}\right\| \leqslant c k^{2-(i+j) / 2}, \quad i, j=0,1 .
$$


Letting $\bar{Q}(z)=1+z^{q / 2}$, we can show that there exist constants $c_{2}>c_{1}>0$ such that $c_{1} \bar{Q}(z) \leqslant Q^{1 / 2}(z) \leqslant c_{2} \bar{Q}(z), \forall z \geqslant 0$. It then follows from a spectral argument that

$$
\left\|\left[\bar{Q}\left(k L_{h}^{n}\right)\right]^{-1} Q^{1 / 2}\left(k L_{h}^{n}\right)\right\| \leqslant c_{2} .
$$

Letting $Q^{1 / 2}=Q^{1 / 2}\left(k L_{h}^{n}\right)$ and $\bar{Q}^{-1}=\left[\bar{Q}\left(k L_{h}^{n}\right)\right]^{-1}$, we have

$$
\begin{aligned}
\left(\left[\mathscr{R}_{h}^{n}-\mathscr{R}_{h}^{n-1}\right] \zeta^{n}, \zeta^{n}\right) & \\
=\sum_{i, j=0}^{1} k^{(i+j) / 2-1}( & \left(T_{h}^{n}\right)^{(1-j) / 2}\left(\mathscr{R}_{h}^{n}-\mathscr{R}_{h}^{n-1}\right)\left(T_{h}^{n}\right)^{(1-i) / 2} \\
& \times\left[\bar{Q}^{-1} Q^{1 / 2}\right]\left(k L_{h}^{n}\right)^{(i(q-1)+1) / 2} Q^{-1 / 2} \zeta^{n}, \\
& {\left.\left[\bar{Q}^{-1} Q^{1 / 2}\right]\left(k L_{h}^{n}\right)^{(j(q-1)+1) / 2} Q^{-1 / 2 \zeta^{n}}\right) . }
\end{aligned}
$$

It then follows from (5.32) and (5.33) that

$$
\begin{aligned}
& \left|\left(\left[\mathscr{R}_{h}^{n}-\mathscr{R}_{h}^{n-1}\right] \zeta^{n}, \zeta^{n}\right)\right| \\
& \quad \leqslant c k\left\{\left\|\left(k L_{h}^{n}\right)^{1 / 2} Q^{-1 / 2} \zeta^{n}\right\|^{2}+\left\|\left(k k L_{h}^{n}\right)^{q / 2} Q^{-1 / 2} \zeta^{n}\right\|^{2}\right\} .
\end{aligned}
$$

Furthermore, for any $\varepsilon>0$, from (4.14),

$$
\begin{aligned}
& \left|\left(\mathscr{R}_{h}^{n} \zeta^{n+1}, \zeta^{n}\right)-\left(\mathscr{R}_{h}^{n} \zeta^{n}, \zeta^{n+1}\right)\right| \\
& \quad=\left|\left(\left[\mathscr{R}_{h}^{n}-r_{h}^{n}\right]\left[\zeta^{n+1}-\zeta^{n}\right], \zeta^{n+1}\right)-\left(\left[\mathscr{R}_{h}^{n}-r_{h}^{n}\right] \zeta^{n+1}, \zeta^{n+1}-\zeta^{n}\right)\right| \\
& \quad \leqslant \varepsilon\left\|\zeta^{n+1}-\zeta^{n}\right\|^{2}+\frac{c}{\varepsilon} k^{2}\left\|\zeta^{n+1}\right\|^{2} .
\end{aligned}
$$

Thus, from (5.29), (5.30), (5.34) and (5.35),

$$
\begin{aligned}
& c(\delta)\left\|\zeta^{n+1}-\zeta^{n}\right\|^{2}+\left(\left[I-\mathscr{R}_{h}^{n}\right] \zeta^{n+1}, \zeta^{n+1}\right) \\
& \leqslant\left(\left[I-\mathscr{R}_{h}^{n-1}\right] \zeta^{n}, \zeta^{n}\right)+c k^{2}\left\|\zeta^{n+1}\right\|^{2} \\
&+c\left\{\left\|\mathscr{R}_{h}^{n} w^{n}-w^{n+1}\right\|^{2}+\left\|\mathscr{R}_{h}^{n} U_{h}^{n}-U_{h}^{n+1}\right\|^{2}\right\} \\
&+c k\left\{\left\|\left(k L_{h}^{n}\right)^{1 / 2} Q^{-1 / 2} \zeta^{n}\right\|^{2}+\left\|\left(k L_{h}^{n}\right)^{q / 2} Q^{-1 / 2} \zeta^{n}\right\|^{2}\right\} .
\end{aligned}
$$

Now with $\bar{\zeta}^{n+1}=\left(k L_{h}^{n}\right)^{1 / 2 \zeta^{n+1}}$ and $\bar{\zeta}^{n}=\left(k L_{h}^{n}\right)^{1 / 2} \zeta^{n}$, we have from (5.15):

$$
\begin{aligned}
& \left(\left[I+\overline{\mathscr{R}}_{h}^{n}\right]\left[\bar{\zeta}^{n+1}-\bar{\zeta}^{n}\right], \bar{\zeta}^{n+1}-\bar{\zeta}^{n}\right)+\left(\left[I-\overline{\mathscr{R}}_{h}^{n}\right] \bar{\zeta}^{n+1}, \bar{\zeta}^{n+1}\right) \\
& =\left(\left[I-\overline{\mathscr{R}}_{h}^{n-1}\right]\left(k L_{h}^{n-1}\right)^{1 / 2} \zeta^{n},\left(k L_{h}^{n-1}\right)^{1 / 2} \zeta^{n}\right) \\
& +\left(\left[I-\overline{\mathscr{R}}_{h}^{n}\right] \bar{\zeta}^{n}, \bar{\zeta}^{n}\right)-\left(\left[I-\overline{\mathscr{R}}_{h}^{n-1}\right]\left(k L_{h}^{n-1}\right)^{1 / 2} \zeta^{n},\left(k L_{h}^{n-1}\right)^{1 / 2} \zeta^{n}\right) \\
& +\left(\overline{\mathscr{R}}_{h}^{n \bar{\zeta}^{n}}, \bar{\zeta}^{n+1}\right)-\left(\overline{\mathscr{R}}_{h}^{n} \bar{\zeta}^{n+1}, \bar{\zeta}^{n}\right) \\
& +2\left(\left(k L_{h}^{n}\right)^{1 / 2}\left[w^{n+1}-\mathscr{R}_{h}^{n} w^{n}+\mathscr{R}_{h}^{n} U_{n}^{n}-U_{h}^{n+1}\right], \bar{\zeta}^{n+1}-\bar{\zeta}^{n}\right) .
\end{aligned}
$$

Now as before, for some $\bar{c}(\delta)>0$,

$$
\left(\left[I+\overline{\mathscr{R}}_{h}^{n}\right]\left[\bar{\zeta}^{n+1}-\bar{\zeta}^{n}\right], \bar{\zeta}^{n+1}-\bar{\zeta}^{n}\right) \geqslant \bar{c}(\delta)\left\|\bar{\zeta}^{n+1}-\bar{\zeta}^{n}\right\|^{2},
$$


and

$$
\left|\left(\overline{\mathscr{R}}_{h}^{n} \bar{\zeta}^{n}, \bar{\zeta}^{n+1}\right)-\left(\overline{\mathscr{R}}_{h}^{n} \bar{\zeta}^{n+1}, \bar{\zeta}^{n}\right)\right| \leqslant \varepsilon\left\|\bar{\zeta}^{n+1}-\bar{\zeta}^{n}\right\|^{2}+\frac{c}{\varepsilon} k^{2}\left\|\bar{\zeta}^{n+1}\right\|^{2}
$$

Furthermore,

$$
\left(\left[I-\overline{\mathscr{R}}_{h}^{n}\right] \bar{\zeta}^{n}, \bar{\zeta}^{n}\right)-\left(\left[I-\overline{\mathscr{R}}_{h}^{n-1}\right]\left(k L_{h}^{n-1}\right)^{1 / 2} \zeta^{n},\left(k L_{h}^{n-1}\right)^{1 / 2} \zeta^{n}\right)=\left(B \bar{\zeta}^{n}, \bar{\zeta}^{n}\right),
$$

where

$$
\begin{aligned}
B= & I-\overline{\mathscr{R}}_{h}^{n}-\left(T_{h}^{n}\right)^{1 / 2}\left(L_{h}^{n-1}\right)^{1 / 2}\left[I-\overline{\mathscr{R}}_{h}^{n-1}\right]\left(L_{h}^{n-1}\right)^{1 / 2}\left(T_{h}^{n}\right)^{1 / 2} \\
= & \left(T_{h}^{n}\right)^{1 / 2}\left(L_{h}^{n}-L_{h}^{n-1}\right)\left(T_{h}^{n}\right)^{1 / 2} \\
& -\left(T_{h}^{n}\right)^{1 / 2}\left(L_{h}^{n-1}\right)^{1 / 2}\left(L_{h}^{n-1}\right)^{1 / 2}\left(r_{h}^{n-1}-\mathscr{R}_{h}^{n-1}\right)\left(T_{h}^{n}\right)^{1 / 2} \\
& +\left(L_{h}^{n}\right)^{1 / 2}\left(r_{h}^{n}-\mathscr{R}_{h}^{n}\right)\left(T_{h}^{n}\right)^{1 / 2}+\left(T_{h}^{n}\right)^{1 / 2}\left(L_{h}^{n-1}-L_{h}^{n}\right) r_{h}^{n-1}\left(T_{h}^{n}\right)^{1 / 2} \\
& +\left(L_{h}^{n}\right)^{1 / 2}\left(r_{h}^{n-1}-r_{h}^{n}\right)\left(T_{h}^{n}\right)^{1 / 2} .
\end{aligned}
$$

By previously used techniques, it can be shown that $\|B\| \leqslant c k$. Thus,

$$
\begin{aligned}
\left|\left(B \bar{\zeta}^{n}, \bar{\zeta}^{n}\right)\right| & =\mid \sum_{i, j=0}^{1}\left(B\left[\bar{Q}^{-1} Q^{1 / 2}\right]\left(k L_{h}^{n}\right)^{i q / 2} Q^{-1 / 2 \bar{\zeta}^{n}},\right. \\
& {\left.\left[\bar{Q}^{-1} Q^{1 / 2}\right]\left(k L_{h}^{n}\right)^{j q / 2} Q^{-1 / 2 \bar{\zeta}^{n}}\right) \mid } \\
& \leqslant c k\left\{\left\|\left(k L_{h}^{n}\right)^{1 / 2} Q^{-1 / 2 \zeta^{n}}\right\|^{2}+\left\|\left(k L_{h}^{n}\right)^{(q+1) / 2} Q^{-1 / 2} \zeta^{n}\right\|^{2}\right\} .
\end{aligned}
$$

Using the last inequality together with (5.36)-(5.38), we get (5.28).

Remark. Inequality (5.28) is adapted to the stability result of Theorem 4.1, in the sense that the last three terms of (5.28) can be shown to match (after dividing by $k$ ) the term

$$
-\frac{\delta}{3}\left(\left[I-r_{h}^{n}\right]\left[I+c^{*} k L_{h}^{n}\right] \zeta^{n}, \zeta^{n}\right)
$$

in (4.9). The corresponding term in (4.16) is $-c_{1} k\left(L_{h}^{n} \zeta^{n}, \zeta^{n}\right)$. Accordingly, by taking $\bar{Q}=I$ in the proof of Proposition 5.4, we obtain the following:

$$
\begin{aligned}
& c_{\delta}\left\|\zeta^{n+1}-\zeta^{n}\right\|_{c^{*}, n+1}^{2}+\left(\left[I-\mathscr{R}_{h}^{n}\right] \zeta^{n+1}, \zeta^{n+1}\right) \\
&+c^{*}\left(\left[I-\overline{\mathscr{R}}_{h}^{n}\right]\left(k L_{h}^{n}\right)^{1 / 2} \zeta^{n+1},\left(k L_{h}^{n}\right)^{1 / 2} \zeta^{n+1}\right) \\
& \leqslant\left(\left[I-\mathscr{R}_{h}^{n-1}\right] \zeta^{n}, \zeta^{n}\right)+c^{*}\left(\left[I-\overline{\mathscr{R}}_{h}^{n-1}\right]\left(k L_{h}^{n-1}\right)^{1 / 2} \zeta^{n},\left(k L_{h}^{n-1}\right)^{1 / 2} \zeta^{n}\right) \\
&+c k^{2}\left\|\zeta^{n+1}\right\|_{c^{*}, n+1}^{2}+c\|\| \mathscr{R}_{h}^{n} w^{n}-w^{n+1} \|_{c^{*}, n+1}^{2} \\
&+c\left\|\mathscr{R}_{h}^{n} U_{h}^{n}-U_{h}^{n+1}\right\|_{c^{*}, n+1}^{2}+c k\left\|\left(k L_{h}^{n}\right)^{1 / 2} \zeta^{n}\right\| .
\end{aligned}
$$

For our next result, we shall require the following condition

$$
(Q-P)(z) \geqslant c\left(z+z^{q}\right) \quad \forall z \geqslant 0 .
$$

Note that from (3.10) and (3.13), this is satisfied for all IRKC methods for which $\gamma_{i} \geqslant 1 / 2, q \geqslant 3$, and $\gamma_{i}>1 / 2, q=1$ or 2 . 
THEOREM 5.2. Suppose the conditions of Theorem 4.3 are satisfied, (3.6) and (5.39) hold and that the eigenvalues of $A$ are real positive. Let $\left\{U_{h}^{n}\right\}_{n=0}^{n^{*}}$ be the sequence of fully discrete approximations generated by the second variant of the base scheme. Then there exist constants $0<c, \varepsilon<1$ such that if

$$
\beta_{n}^{2} \leqslant c \varepsilon t_{n-\sigma}
$$

then

$$
\max _{0 \leqslant n \leqslant n^{*}}\left\|U_{h}^{n}-w^{n}\right\|_{c^{*}, n} \leqslant c \mathscr{E}\left\|u^{0}\right\|_{\mu}
$$

and

$$
\max _{0 \leqslant n \leqslant n^{*}}\left\|U_{h}^{n}-u^{n}\right\| \leqslant c \mathscr{E}\left\|u^{0}\right\|_{\mu} .
$$

Proof. Let $\varepsilon>0$ be a constant to be appropriately chosen below. Multiplying (5.21) and (5.28) with $k^{-1} \varepsilon t_{n-\sigma}$ and using (4.9), we get

$$
\begin{aligned}
& \left(1-c k \varepsilon t_{n-\sigma}\right)\left\|\zeta^{n+1}\right\|_{c^{*}, n+1}^{2}+c_{\delta} k^{-1} \varepsilon t_{n-\sigma}\left\|\zeta^{n+1}-\zeta^{n}\right\|_{c^{*}, n+1}^{2} \\
& +k^{-1} \varepsilon t_{n-\sigma}\left\{\left(\left[I-\mathscr{R}_{h}^{n}\right] \zeta^{n+1}, \zeta^{n+1}\right)\right. \\
& \left.+\left(\left[I-\overline{\mathscr{R}}_{h}^{n}\right]\left(k L_{h}^{n}\right)^{1 / 2} \zeta^{n+1},\left(k L_{h}^{n}\right)^{1 / 2} \zeta^{n+1}\right)+\left\|G^{n}\right\|_{c^{*}, n}^{2}\right\} \\
& \leqslant(1+\tilde{c} k)\left\|\zeta^{n}\right\|_{c^{*}, n}^{2}+c k \beta_{n}^{2}\left(1+\varepsilon t_{n-\sigma}\right) \sum_{l=1}^{\sigma+1}\left\|\zeta^{n-l}\right\|_{c^{*}, n-l}^{2} \\
& +\left[\varepsilon k^{-1} t_{n-\sigma-1}+\varepsilon\right]\left\{\left(\left[I-\mathscr{R}_{h}^{n-1}\right] \zeta^{n}, \zeta^{n}\right)\right. \\
& \left.+\left(\left[I-\overline{\mathscr{R}}_{h}^{n-1}\right]\left(k L_{h}^{n-1}\right)^{1 / 2} \zeta^{n},\left(k L_{h}^{n-1}\right)^{1 / 2} \zeta^{n}\right)\right\} \\
& +c k^{-1} \beta_{n}^{2}\left(1+\varepsilon t_{n-\sigma}\right)\left\{\sum_{l=0}^{\sigma}\left\|\zeta^{n-l}-\zeta^{n-l-1}\right\|_{c^{*}, n-l}^{2}+\sum_{l=1}^{\sigma+1}\left\|G^{n-l}\right\|_{c^{*}, n-l}^{2}\right\} \\
& +c k\left(1+\beta_{n}^{2}\right)\left(1+\varepsilon t_{n-\sigma}\right) \mathscr{E}^{2}\left\|u^{0}\right\|_{\mu}^{2}-\frac{\delta}{3}\left(\left[I-r_{h}^{n}\right]\left[I+c^{*} k L_{h}^{n}\right] \zeta^{n}, \zeta^{n}\right) \\
& +c \varepsilon t_{n-\sigma}\left\{\left\|\left(k L_{h}^{n}\right)^{1 / 2} Q^{-1 / 2} \zeta^{n}\right\|^{2}\right. \\
& \left.+\left\|\left(k L_{h}^{n}\right)^{q / 2} Q^{-1 / 2} \zeta^{n}\right\|^{2}+\left\|\left(k L_{h}^{n}\right)^{(q+1) / 2} Q^{-1 / 2} \zeta^{n}\right\|^{2}\right\},
\end{aligned}
$$

$\sigma+1 \leqslant n \leqslant n^{*}-1$. Here $\tilde{c}$ is as in (4.9), and we have assumed $\beta_{n} \geqslant c k$. Now, from (5.39),

$$
\begin{aligned}
& \left(\left[I-r_{h}^{n}\right]\left[I+c^{*} k L_{h}^{n}\right] \zeta^{n}, \zeta^{n}\right) \\
& =\left(\left[Q\left(k L_{h}^{n}\right)-P\left(k L_{h}^{n}\right)\right]\left[I+c^{*} k L_{h}^{n}\right] Q^{\left.-1 / 2 \zeta^{n}, Q^{-1 / 2} \zeta^{n}\right)}\right. \\
& \geqslant c\left\{\left\|\left(k L_{h}^{n}\right)^{1 / 2} Q^{-1 / 2} \zeta^{n}\right\|^{2}+\left\|\left(k L_{h}^{n}\right)^{q / 2} Q^{-1 / 2 \zeta^{n}}\right\|^{2}\right. \\
& \left.+\left\|\left(k L_{h}^{n}\right)^{(q+1) / 2} Q^{-1 / 2 \zeta^{n}}\right\|^{2}\right\} .
\end{aligned}
$$


As in the proof of Proposition 5.4, we can show that

$$
\begin{gathered}
\left(\left[I-\mathscr{R}_{h}^{n-1}\right] \zeta^{n}, \zeta^{n}\right)+\left(\left[I-\overline{\mathscr{R}}_{h}^{n-1}\right]\left(k L_{h}^{n-1}\right)^{1 / 2} \zeta^{n},\left(k L_{h}^{n-1}\right)^{1 / 2} \zeta^{n}\right) \\
=\left(\left[I-r_{h}^{n}\right]\left[I+k L_{h}^{n}\right] \zeta^{n}, \zeta^{n}\right)+\psi^{n},
\end{gathered}
$$

where $\psi^{n}$ satisfies

$$
\left|\psi^{n}\right| \leqslant c k\left|\left\|\zeta^{n} \mid\right\|_{c^{*}, n} .\right.
$$

Choosing $\varepsilon$ and $\beta_{n}^{2}$ so that $\beta_{n}^{2} \leqslant c \varepsilon t_{n-\sigma} \leqslant c \varepsilon t^{*} \leqslant c \delta$ for some $c<1$, and summing (5.43) over $n=\sigma+1, \ldots, N-1$ for some $\sigma+2 \leqslant N \leqslant n^{*}$, we get, using (5.44), (5.45) and (5.46),

$$
\begin{aligned}
\left\|\zeta^{N}\right\|_{c^{*}, N}^{2} \leqslant & c\left\|\zeta^{\sigma+1}\right\|_{c^{*}, \sigma+1}^{2}+c k \sum_{j=0}^{N-1}(\tilde{c}+c)\left\|\zeta^{j}\right\|_{c^{*}, j}^{2} \\
& +c \sum_{j=1}^{\sigma+1}\left\|\zeta^{j}-\zeta^{j-1}\right\|_{c^{*}, j}^{2}+c \sum_{j=0}^{\sigma}\left\|G^{j}\right\|_{c^{*}, j}^{2} \\
& +c k \sum_{j=\sigma+1}^{N-1} \mathscr{E}^{2}\left\|u^{0}\right\|_{\mu}^{2}, \quad N=\sigma+2, \ldots, n^{*} .
\end{aligned}
$$

Using a discrete version of Gronwall's Lemma, we get

$$
\begin{array}{r}
\left\|\zeta^{N}\right\|^{2} \leqslant c\left\{\sum_{j=0}^{\sigma+1}\left\|\zeta^{j}\right\|_{c^{*}, j}^{2}+\sum_{j=0}^{\sigma}\|\| G^{j}\left\|_{c^{*}, j}^{2}+\mathscr{E}^{2}\right\| u^{0} \|^{2}\right\} \\
N=\sigma+2, \ldots, n^{*} .
\end{array}
$$

Finally, since

$$
\left\{\left\|\zeta^{l}\right\|_{c^{*}, l},\left\|G^{\prime}\right\|_{c^{*}, l}\right\}_{l=0}^{\sigma+1} \leqslant c \mathscr{E}^{\prime=u^{0}} \|_{\mu},
$$

(5.41) and (5.42) follow from (5.47) and (5.48).

Remarks. (i) For simplicity, we have assumed that $\left\{U_{h}^{n}\right\}_{n=0}^{\sigma+1}$ are generated using the first variant. In fact, one can use the second variant itself by suitably changing the coefficients in (5.19); and, in general, any method for which (5.48) holds.

(ii) The inequality $\beta_{n}^{2} \leqslant c \varepsilon t_{n-\sigma}$ implies an $O\left(\ln k^{-1}\right)$ amount of work in the earlier steps. However, it can be shown that the average work per step is independent of $k$ (cf. [5]).

Department of Mathematics The University of Tennessee Knoxville, Tennessee 37996

1. O. AxELSSON, "Solution of linear systems of equations: Iterative methods," in Sparse Matrix Techniques (V. A. Barker, ed.), Lecture Notes in Math., Vol. 572, Springer-Verlag, Berlin, Heidelberg and New York, 1976, pp. 1-51.

2. G. A. Baker, J. H. Bramble \& V. ThomEe, "Single step Galerkin approximations for parabolic problems," Math. Comp., v. 31, 1977, pp. 818-847.

3. L. A. BALES, "Semidiscrete and single step fully discrete approximations for second order hyperbolic equations with time-dependent coefficients," Math. Comp., v. 43, 1984, pp. 383-414.

4. L. A. Bales, O. A. Karakashian \& S. M. Serbin, "On the $A_{0}$-stability of rational approximations to the exponential function with only real poles." (Submitted for publication.)

5. J. H. Bramble \& P. H. Sammon, "Efficient higher order single-step methods for parabolic problems: Part I," Math. Comp., v. 35, 1980, pp. 655-677. 
6. K. Burrage, "Efficiently implementable algebraically stable Runge-Kutta methods," SIAM J. Numer. Anal., v. 19, 1982, pp. 245-258.

7. K. Burrage \& J. C. Butcher, "Stability criteria for implicit Runge-Kutta methods," SIAM J. Numer. Anal., v. 16, 1979, pp. 46-57.

8. J. C. Butcher, “Implicit Runge-Kutta processes,” Math. Comp., v. 18, 1964, pp. 50-64.

9. J. C. ButCher, "A stability property of implicit Runge-Kutta methods," BIT, v. 15, 1975, pp. $358-361$.

10. J. C. ButCHER, “On the implementation of implicit Runge-Kutta methods,” $B I T$, v. 16, 1976, pp. 237-240.

11. M. Crouzeix, Sur l'Approximation des Équations Différentielles Opérationnelles Linéaires par des Méthodes de Runge-Kutta, Thèse, Université de Paris VI, 1975.

12. M. Crouzeix, "Sur la B-stabilité des méthodes de Runge-Kutta," Numer. Math., v. 32, 1979, pp. 75-82.

13. J. Douglas, JR., T. Dupont \& R. Ewing, "Incomplete iterations for time-stepping a Galerkin method for a quasilinear parabolic problem," SIAM J. Numer. Anal., v. 16, 1979, pp. 503-522.

14. J. L. Lions \& E. Magenes, Nonhomogeneous Boundary Value Problems and Applications, Vol. II, Springer-Verlag, New York, 1973.

15. S. P. N $\phi$ RSETT, “One step methods of Hermite type for numerical integration of stiff systems," $B I T$, v. 14,1974, pp. $63-77$.

16. S. P. NøRSETt \& G. WANNER, "The real-pole sandwich for rational approximations and oscillation equations," BIT, v. 19, 1979, pp. 79-94.

17. P. H. Sammon, Approximations for Parabolic Equations with Time-Dependent Coefficients, Ph.D. Thesis, Cornell University, Ithaca, N.Y., 1978.

18. P. H. SAmmon, Convergence Estimates for Semidiscrete Parabolic Approximations, Mathematics Research Center Technical Survey Report No. 2053, 1980.

19. A. WOLFBRANDT, "A note on a recent result of rational approximations to the exponential function," $B I T$, v. 17,1977 , pp. 367-368. 